\title{
Characterization of the subunit composition and structure of native adult
}

\section{glycine receptors} 75390, USA

*Correspondence: Weiwei.Wang@UTSouthwestern.edu

\section{SUMMARY}

The strychnine-sensitive pentameric Glycine Receptor (GlyR) mediates fast inhibitory

11 neurotransmission in the mammalian nervous system. Only heteromeric GlyRs mediate synaptic

12 transmission, since they contain the $\beta$ subunit that permits clustering at the synapse through its

13 interaction with scaffolding proteins. Here we show that $\alpha 2$ and $\beta$ subunits assemble with an

14 unexpected 4:1 stoichiometry to produce GlyR with native electrophysiological properties. We

15 determined structures in multiple functional states at $3.6-3.8 \AA$ resolutions and show $\alpha 2 \beta$ GlyR assembly mechanism. Furthermore, we show that one single $\beta$ subunit in each GlyR gives rise to

17 the characteristic electrophysiological properties of heteromeric GlyR, while more $\beta$ subunits

18 renders GlyR non-conductive. A single $\beta$ subunit ensures a univalent GlyR-scaffold linkage,

19 which means the scaffold alone regulates the cluster properties.

KEYWORDS: heteromeric glycine receptor, Cryo-EM structure, stoichiometry, planar bilayer

21 recording, stepwise photobleaching 


\section{INTRODUCTION}

2 Nervous system function depends on electrical signal transmission across neuronal cells. At chemical synapses, electrical signals are transmitted through the release of neurotransmitters from the pre-synaptic neuron and resultant activation of the cognate neurotransmitter receptors in the post-synaptic neuron (Eccles, 1982). The neurotransmitter glycine mediates fast inhibitory neurotransmission through activating the strychnine-sensitive glycine receptors (GlyRs) at inhibitory synapses (Moss and Smart, 2001; Pfeiffer and Betz, 1981; Werman et al., 1967). GlyRs are expressed in both the central and peripheral nervous systems(Lynch, 2004). Defects in GlyRs cause the neurological disorder hyperekplexia (Bode and Lynch, 2014) and are implicated in autism spectrum disorders (Yu et al., 2013). GlyRs have also been found in the visual system (Wassle et al., 2009) and identified as possible therapeutic targets for inflammatory pain (Harvey et al., 2004; Lynch and Callister, 2006).

GlyRs are members in the ionotropic pentameric Cys-loop receptor superfamily. There are two major types of GlyRs, homomeric GlyRs composed of only $\alpha$ subunits and heteromeric GlyRs consisting of both $\alpha$ and $\beta$ subunits. Extensive study on the homomeric GlyRs (Du et al., 2015; Huang et al., 2015; Kumar et al., 2020; Yu et al., 2021) and related Cys-loop receptors (Phulera et al., 2018; Rahman et al., 2020; Zhu et al., 2018) provided little information on the architecture and working mechanism of native adult GlyRs for the following reasons. First, homomeric GlyRs are found almost exclusively during early development while heteromeric GlyRs is the predominantly form in adult animals (Malosio et al., 1991; Weltzien et al., 2012). Second, heteromeric GlyRs exhibit very different electrophysiological properties, which is solely determined by the $\beta$ subunit that occur only in the heteromeric GlyRs (Bormann et al., 1993; Lynch, 2009; Pribilla et al., 1992). Third, heteromeric GlyRs form high-density clusters at post- 
1 synaptic membrane and mediate synaptic transmission while homomeric GlyRs do not localize at synapses (Kirsch et al., 1993; Specht et al., 2013). This is because homomeric GlyRs do not contain the $\beta$ subunit with which post-synaptic scaffolding protein gephyrin directly associates

4 (Meyer et al., 1995). controversial for decades (Durisic et al., 2012; Grudzinska et al., 2005; Kuhse et al., 1993; Langosch et al., 1988; Patrizio et al., 2017; Yang et al., 2012). The number and spatial arrangement of $\beta$ subunits in each GlyRs are central questions in synaptic transmission since they impact how heteromeric GlyR clusters with multivalent scaffolding protein gephyrin (Sander et al., 2013; Sola et al., 2004) and regulate synaptic plasticity (Citri and Malenka, 2008; Zacchi et al., 2014; Zeng et al., 2018). Here, we characterized the function of heteromeric $\alpha 2 \beta$ GlyR both in cells and in total reconstitution systems. A combination of biochemical and structural analyses revealed the molecular mechanism underlying the assembly of $\alpha 2 \beta$ GlyR consisting four $\alpha 2$ and one $\beta$ subunit, as well as how such molecular composition gives rise to native GlyR electrophysiological characteristics, including glycine activation, single-channel conductance and picrotoxin (PTX) inhibition. We further show how $\alpha 2 \beta$ GlyR containing more than one $\beta$ subunit does not conduct $\mathrm{Cl}^{-}$upon glycine activation. Our findings lead to the conclusion that a 4:1 $\alpha 2: \beta$ stoichiometry accounts for the native physiology of heteromeric GlyRs.

\section{RESULTS}

\section{Invariant $\alpha 2 \beta$ GlyR subunit stoichiometry}

21 We produced heteromeric $\alpha 2 \beta$ GlyR through co-expressing the $\alpha 2$ and the $\beta$ subunits in HEK293

22 cells for structural and functional characterization. We have identified $\alpha 2 \beta$ GlyR constructs,

$23 \alpha 2 \mathrm{em}$ and $\beta$ em (see methods for details, denoted here $\alpha 2$ and $\beta$ unless otherwise indicated), that 
1 exhibited both good physiological function (Figure S1, A and B) and biochemical behavior,

2 allowing unambiguous characterization of the molecular composition. Depending on the

3 expression levels of each subunit, homomeric $\alpha 2$ GlyR, heteromeric $\alpha 2 \beta$ GlyR, or a mixture of

4 both may be produced. We used whole-cell patch-clamp electrophysiology to characterize the

5 GlyRs residing in the plasma membrane as a function of expression levels.

6 Homomeric GlyRs are inhibited by the convulsant alkaloid, pore-blocking toxin picrotoxin

7 (PTX) in the micro-molar range while native heteromeric GlyRs are more resistant with an over

8 10-fold higher $\mathrm{IC}_{50}$ (Pribilla et al., 1992; Shan et al., 2001). When transfected with only $\alpha 2$,

9 homomeric GlyRs exhibited high affinity to PTX with an $\mathrm{IC}_{50}$ of $\sim 2.4 \mu \mathrm{M}$ (Figure $1 \mathrm{~A}$ and $1 \mathrm{C}$

10 black, Figure S1D) (Pribilla et al., 1992; Yang et al., 2007). When a moderate amount of the $\beta$

11 subunit was co-expressed, at 1:1 $\alpha 2: \beta$ ratio (transfected DNA amounts, Figure $\mathrm{S} 1 \mathrm{C}$ ), the $\mathrm{IC}_{50}$

12 increased to $\sim 11 \mu \mathrm{M}$ (Figure $1 \mathrm{C}$ green), indicating that a mixture of $\alpha 2$ and $\alpha 2 \beta$ GlyR were

13 produced. When an excess of the $\beta$ subunit was expressed at 1:3 $\alpha 2: \beta$ ratio, PTX $\mathrm{IC}_{50}$ increased

14 further to $\sim 30 \mu \mathrm{M}$ (Figure 1, B and C blue). However, a greater excess of the $\beta$ subunit (at 1:10

$15 \alpha 2: \beta$ ratio) did not result in further change (Figure $1 \mathrm{C}$ red).

16 Unlike PTX, neurotransmitter glycine binds to the GlyR extracellular domain, distant from the

17 ion conduction pore. Based on the difference in its affinity for homomeric and heteromeric GlyR

18 (Grudzinska et al., 2005; Pribilla et al., 1992), we used glycine as an additional probe of the

19 formation of $\alpha 2 \beta$ GlyR (Figure S1E). When only $\alpha 2$ GlyR was expressed, a $\sim 100 \mu \mathrm{M} \mathrm{EC}_{50}$

20 (Figure 1, D and F black) was observed. With both the 1:3 and 1:10 $\alpha 2: \beta$ ratio, the $\mathrm{EC}_{50}$

21 decreased to $\sim 60 \mu \mathrm{M}$ (Figure $1, \mathrm{E}$ and $\mathrm{F}$ blue, red). These numbers are within the range of

22 reported glycine affinities for $\alpha 2$ and $\alpha 2 \beta$ GlyRs (Grudzinska et al., 2005; Mohammadi et al.,

23 2003; Pribilla et al., 1992). Clearly, the change in glycine sensitivity followed a similar trend as 
1 that of PTX - the effects of $\beta$ subunit expression saturate at 1:3 $\alpha 2: \beta$ ratio. This trend has two implications: first, at a 1:3 $\alpha 2: \beta$ ratio, sufficient $\beta$ subunit is expressed to make $\alpha 2 \beta$ GlyR the predominant form. Second, the number of $\beta$ subunits in each $\alpha 2 \beta$ GlyR did not further increase when the $\alpha 2: \beta$ ratio was changed from $1: 3$ to $1: 10$, since the incorporation of additional $\beta$ subunits should further change the PTX and glycine affinities (Pribilla et al., 1992; Shan et al., $62001)$

We further used Fluorescence Size Exclusion Chromatography (FSEC) to test whether the number of $\beta$ subunit in each $\alpha 2 \beta$ GlyR stayed the same irrespective of expression levels of $\alpha 2$ and $\beta$ subunits. Since the $\beta$ subunit has a higher molecular weight than that of $\alpha 2$, changes in $\alpha 2: \beta$ stoichiometry should result in changes of elution volumes. As control experiments, we coexpressed $\alpha 2$, and the higher molecular weight fluorescent fusion $\alpha 2$-Tsi-GFP (see methods), over a wide range of expression ratios (virus MOI, see methods) (Morales-Perez et al., 2016). A clear shift in the peak elution volume was observed (Figure $1 \mathrm{G}$ and Figure S1F, left). This finding supports that $\alpha$ subunits are able to form GlyRs with arbitrary stoichiometries (Griffon et al., 1999; Kuhse et al., 1993). In contrast, when co-expressing $\alpha 2$ and $\beta$, the peak elution volume did not change over the same range of virus ratios (Figure $1 \mathrm{H}$ and Figure $\mathrm{S} 1 \mathrm{~F}$, right). These observations point to an invariant subunit stoichiometry irrespective of expression levels of $\alpha 2$ and $\beta$, consistent with our and previously reported electrophysiology data (Durisic et al., 2012; Kuhse et al., 1993). Clearly, GlyRs are unlike other members of the Cys-loop receptor family in which variability in stoichiometry has been shown (Morales-Perez et al., 2016).

\section{$21 \quad$ Planar lipid bilayer electrophysiology} been characterized (Cascio et al., 2001), functional reconstitution of heteromeric GlyR has not 
been reported. We employed the planar lipid bilayer method to characterize the electrophysiology of purified $\alpha 2 \beta$ GlyR in vitro (Figure 2A) (Mueller et al., 1962; Wang et al., 2014). In this method, a synthetic bilayer was created using the painting method across a $\sim 100$ $\mu \mathrm{m}$ hole. Purified $\alpha 2$ ( $\alpha 2$-Tsi-GFP) and $\alpha 2 \beta$ (expressed with 1:3 $\alpha 2: \beta$ ratio) GlyRs were reconstituted into proteoliposomes and subsequently incorporated into the synthetic membrane

through salt-assisted spontaneous fusion. GlyR electrophysiological properties were thus characterized in a chemically defined environment.

The $\alpha 2$ GlyR exhibited rare spontaneous openings in the absence of ligands (Figure 2C left). When $200 \mu \mathrm{M}$ glycine was applied to the same membrane, robust channel activation was observed (Figure 2C middle). This observation is consistent with the low 'basal' activity and strong glycine activation found in cellular membranes (Schmieden et al., 1989; Sontheimer et al., 1989) (See Figure 1). A histogram of the current levels (Figure 2E black) led to the identification of a $75 \mathrm{pS}$ single-channel conductance. This value is consistent with one major conductance observed in cells ( 70 pS) (Bormann et al., 1993; Cascio et al., 1993; Lynch, 2009). Subsequent application of $10 \mu \mathrm{M}$ competitive antagonist strychnine(Becker et al., 1988) on the same side of the membrane almost completely inhibited $\alpha 2$ GlyR activity (Figure $2 \mathrm{C}$ right). Very similar pharmacology - low basal activity (Figure 2D left), strong glycine activation (Figure 2D middle) and strychnine inhibition (Figure 2D right) - was observed for the $\alpha 2 \beta$ GlyR. However, the $\alpha 2 \beta$ GlyR had a much lower single-channel conductance of $\sim 48 \mathrm{pS}$ (Figure 2E blue), which recapitulates the major conductance of heteromeric GlyRs both heterologously expressed and in native adult tissues ( $\sim 50$ pS) (Ali et al., 2000; Bormann et al., 1993; Lynch, 2009; Takahashi et al., 1992). The single channel conductance of both $\alpha 2$ and $\alpha 2 \beta$ GlyRs remained constant in bilayers made with either polar lipids extracted from brain tissues or an artificial mimic (see 
1 Methods), indicating minimal lipid effects in these mixtures. The uniformity of single channel conductance (Figure 2E) further confirms a homogeneous subunit stoichiometry of the purified $\alpha 2 \beta$ GlyR, since the unique sequence features in the M2 helix of the $\beta$ subunit has been shown to alter GlyR conductance (Bormann et al., 1993). Therefore, based on the similarity of its electrophysiological properties to native heteromeric GlyRs(Ali et al., 2000; Bormann et al., 1993; Lynch, 2009; Takahashi et al., 1992), we believe this purified $\alpha 2 \beta$ GlyR is likely to be the dominant, physiological form of $\alpha 2 \beta$ GlyR.

\section{4:1 $\alpha 2: \beta$ GlyR structures in the closed, desensitized and open states}

The $\alpha 2 \beta$ GlyR structures were resolved in complex with the neurotransmitter glycine, as well as a competitive antagonist strychnine in saposin nanodiscs (Flayhan et al., 2018). Reconstructed cryo-EM maps contained densities of $\alpha 2 \beta$ GlyR (Figure 3A grey), as well as a single extra satellite near the intracellular side (Figure 3A green) resulting from the GFP fusion in the intracellular M3-M4 loop of the $\beta$ subunit (see methods for details), suggesting that each GlyR contains only one $\beta$ subunit. Within a mask excluding this satellite density (Figure 3A translucent blue surface), the $\alpha 2 \beta$ GlyR maps reached overall resolutions of $3.6-3.8 \AA$ (Figure S2), allowing for unambiguous amino acid registration for the majority of the receptor (Figure S3). One $\beta$ subunit (Figure 3B blue) and four $\alpha 2$ subunits (Figure 3B grey) were identified in each pentameric $\alpha 2 \beta$ GlyR in the atomic models, resulting in an unexpected but unequivocal 4:1 subunit stoichiometry.

Two structural classes were present in the GlyR-strychnine complex (Figure S2A). In both classes, the ion-conduction pores were tightly constricted near the 9' amino acid residues $\alpha 2$ : L268 and $\beta$ : L285 (Figure 3, D and E), too narrow ( $<1.8 \AA$ radii) for even non-hydrated $\mathrm{Cl}^{-}$to pass through (Figure 3C orange). The antagonist strychnine was present in all 5 neurotransmitter 
binding pockets in both structures (Figure S4, A and B left). These binding pockets are in an

2 apo/resting conformation where the loop Cs are in the "uncapped" configuration (Yu et al., 2021)

3 (Figure S5, G and I blue). The differences between these two structures are manifested in the

4 transmembrane domain (TMD) near the intracellular leaflet. One class (closed 1) is pseudo-5-

5 fold symmetrical across the whole receptor (Figure S4, B and C), while the other class (closed 2)

6 has the $\beta$ TM2 tilted toward the ion conduction pore and one of the $\alpha 2$ TM2 tilted out (Figure

7 S4D). Since both structures exhibit functional characteristics of a closed conduction pore, we

8 believe they represents two possible sub-conformations in the closed state of heteromeric GlyR.

9 In the $\alpha 2 \beta$ GlyR-glycine complex, we also identified two distinct structural classes (Figure S2H).

10 In both classes, the conduction pores near the 9' position widened and no longer cause

11 constriction as in the GlyR-strychnine structures. The radii were larger than $1.8 \AA$ (Figure $3 \mathrm{C}$

12 black) that allow partially hydrated $\mathrm{Cl}^{-}$to pass through. In one class, the ion conduction pore

13 narrowed near the -2' amino acid residues $\alpha 2$ : P257 and $\beta$ : A274, resulting in a new constriction

14 near the intracellular side (Figure 3F). Glycine densities were observed in all the 5

15 neurotransmitter binding pockets (Figure S4B middle) that are in the agonist-bound

16 conformation (Figure S5, D-F). These features suggest that this structural class represents the

17 desensitized functional state. In contrast, an asymmetrical widening near -2' position in the other

18 structural class removed constriction (Figure $3 \mathrm{G}$ and Figure S4F). The resultant pore radii were

19 larger than $1.8 \AA$ all across (Figure 3C). All 5 neurotransmitter binding pockets were in the

20 agonist-bound conformation even though clear densities of glycine were observed for only 3

21 (Figure S4B right). Since there is no constriction near the 9' or the -2' positions and the pore size

22 allows for partially hydrated $\mathrm{Cl}^{-}$to pass through, similar to homomeric GlyRs in the open state 
1 (Kumar et al., 2020; Yu et al., 2021), we believe this class represents a structure of $\alpha 2 \beta$ GlyR in the open functional state.

\section{Structural determinants of $\alpha 2 \beta$ GlyR assembly and electrophysiology}

4 The $\alpha 2 \beta$ GlyR shares similar overall structures with homomeric GlyRs (Du et al., 2015; Kumar et al., 2020; Yu et al., 2021). The constriction sites in the ion conduction pathway, 9' in the

6 closed state and $-2^{\prime}$ in the desensitized state, are similar (Figure 3). In addition, the neurotransmitter binding sites are highly conserved across the $\alpha / \beta, \beta / \alpha$ and $\alpha / \alpha$ interfaces and exhibit similar structural changes upon agonist binding (Figure S5). These conservation in binding sites explain the small difference in glycine affinity between $\alpha 2 \beta$ and $\alpha 2$ GlyRs (Figure 1F) (Grudzinska et al., 2005; Pribilla et al., 1992). Despite apparent similarities, several features

11 in the $\beta$ subunit give rise to the unique properties of native GlyRs. sequence homology, two N-glycosylation sites have been predicted (Griffon et al., 1999; Schaefer et al., 2018). $\beta$ :N220 is specific to the $\beta$ subunit and located to the N-terminus of loop C, closer to the neurotransmitter binding pocket compared with $\alpha 2$ (Figure 4A, Figure S3). The $\beta: \mathrm{N} 220 \mathrm{~A}$ mutation abolished glycosylation and prevented proper assembly of the $\alpha 2 \beta$ GlyR (Figure S5J). $\beta$ :N36 was believed homologous to $\alpha 2$ :N45 (Griffon et al., 1999; Schaefer et al., 2018) but turned out to locate near the vestibule (Figure 4A and B). Although the resolution was not sufficient for modeling, density maps show that the glycan extends into the vestibule (Figure 4B). $\beta$ :N36A mutation also prevented expression of the $\alpha 2 \beta$ GlyR (Figure S5J), suggesting its essential role in heteromeric GlyR assembly. Glycosylation at this site is likely a reason for the invariant 4:1 $\alpha 2: \beta$ stoichiometry since the vestibule is not large enough to accommodate more than one $\beta$ :N36 glycan. 
1 The unique amino-acid composition of the pore-lining TM2 of $\beta$ subunit (Figure S6) resulted in

electrophysiological properties specific to heteromeric $\alpha 2 \beta$ GlyR. First, two negatively-charged amino-acid residues, $\beta$ :E297 and $\beta$ :E290, poised near the extracellular side of transmembrane pore. The resultant negative electrostatic potential (Figure 4C) most likely reduced the local concentration of $\mathrm{Cl}^{-}$, decreasing ion conductance. Indeed, the lower single-channel conductance of heteromeric GlyRs (Figure 2E) compared with homomeric GlyRs has been functionally attributed to these residues (Bormann et al., 1993). Second, the bulky side chain of 6' $\beta: F 282$ narrows the pore radii in all functional states compared with homomeric GlyRs (Figure 4, C and D). This explains why heteromeric GlyRs exhibit much lower affinity to the pore blocking PTX: PTX binds near 6' position (Kumar et al., 2020) and $\beta: F 282$ side chain constitutes steric hindrance. Supporting this conclusion, mutating $\beta: F 282$ to amino acids with smaller side-chains rendered high-affinity blocking of heteromeric GlyRs just like with homomeric GlyRs (Pribilla et al., 1992).

\section{Only 4:1 $\alpha 2: \beta$ GlyR conducts}

The negative charges of $\beta: E 297$ and $\beta: E 290$ and reduced pore radii due to the bulky $\beta: F 282$ predict that heteromeric GlyRs containing more than one $\beta$ subunit should be impaired in $\mathrm{Cl}^{-}$ conduction. A hypothetical model of $(\alpha 2)_{3} \beta_{2}$ was constructed based on our open/desensitized structures using a $\alpha 2-\beta-\alpha 2-\beta-\alpha 2$ (meta) configuration where two conduction barriers formed (Figure 4E). First, negative electrostatic potentials in the pore near $\beta$ :E297 and $\beta$ :E290 indicate greatly reduced local $\mathrm{Cl}^{-}$concentration as well as raised electrostatic barrier. Second, the two $\beta:$ F282 sidechains resulted in tight constriction $(<1.8 \AA)$ even in the open/desensitized states. To test whether heteromeric GlyR with more than one $\beta$ subunit is indeed deficient in $\mathrm{Cl}^{-}$ conduction, we generated a chimeric construct, $\beta$ chim, by combining $\alpha 2-\mathrm{ECD}$ and $\beta-\mathrm{TMD}$, to 
overcome the invariant $4: 1$ stoichiometry in wild-type $\alpha 2 \beta$ GlyR. The elution peak of $\alpha 2: \beta \mathrm{chim}$ exhibited a left-shifting trend as $\alpha 2: \beta c h i m$ expression ratios changed from 1:3 (Figure 4F solid blue) to 1:10 (Figure 4F solid red), and to 0:1 (Figure 4F solid green), suggesting arbitrary $\alpha 2: \beta c h i m$ stoichiometry and homomeric $\beta$ chim GlyR formation.

Although $\alpha 2$ and $\beta$ chim formed GlyR with arbitrary stoichiometry biochemically, only the $4: 1$ configuration conducted $\mathrm{Cl}^{-}$. First, the amplitudes of glycine-activated currents decreased as more $\beta$ chim is expressed with respect to $\alpha 2$ (Figure $4 \mathrm{G}, 1: 3$ and 1:10). When only the $\beta$ chim is expressed, glycine elicited no current (Figure 4G, 0:1). The reduction in current is a result of GlyR being non-conductive when 2 or more $\beta$ chim are incorporated, for the following reasons. First, small change in expression levels $(<4$ folds, Figure $4 \mathrm{~F})$ does not account for decimated current levels ( $>1000$ folds). Second, PTX affinities stayed the same as native heteromeric GlyRs in all expression levels (Figure 4H). Since the incorporation of each $\beta$ chim contributes equal amount of steric hindrance at $\beta$ :F282 to destabilizing PTX binding, a same PTX affinity indicates a fixed number (1) of $\beta$ subunit in each conductive GlyR. Higher $\beta$ chim expression levels drive the population of the only conductive species, $4: 1 \alpha 2: \beta \operatorname{chim}$ GlyR, to other stoichiometries, therefore decreasing total measurable current. The "dominant negative" effect reported earlier of a similar chimeric $\beta$ construct also supports this conclusion (Kuhse et al., 1993).

\section{$\alpha 2 \beta$ GlyR distribution in the cellular membrane}

Single-molecule counting allows quantification of membrane proteins in native cell membranes (Patrizio and Specht, 2016; Schmidt et al., 1996). Most research using this method suggested 2 or $3 \beta$ subunits in each $\alpha 1 \beta$ and $\alpha 3 \beta$ GlyRs (Durisic et al., 2012; Patrizio et al., 2017). Since we have already demonstrated an invariant 4:1 $\alpha 2: \beta$ stoichiometry for the $\alpha 2 \beta$ GlyR, this method allowed us to understand how $\alpha 2 \beta$ GlyR is distributed in cell membranes. 
1 One or two homomeric $\alpha 2$ GlyRs were observed in each diffraction limited fluorescent spot

2 ( 250 nm). We used an $\alpha 2$-GFP fusion (see methods), which is known to form pentameric GlyR

3 (Du et al., 2015; Huang et al., 2015; Langosch et al., 1988), to determine the probability, $p_{m}$, of

4 GFP being mature and fluorescent under our expression conditions. In 2295 diffraction-limited

5 fluorescent spots, many had photobleaching steps ranging from 1 to 5 (Figure 5, A and C) while

6 a significant number had between 6 and 10 steps (Figure 5, B and C), suggesting the presence of

7 two $\alpha 2$ GlyRs in some spots. Using a dual-binomial model in data fitting (Figure 5C), we derived

8 a $p_{m}$ of $\sim 62 \%$, with $67 \%$ of the spots containing 1 and $33 \%$ of the spots containing $2 \alpha 2$-GFP

9 GlyRs. Coincident with our findings, multiple $\alpha 1$ GlyRs being present in a single spot has also

10 been reported (McGuire et al., 2012).

11 Up to three $\alpha 2 \beta$ GlyRs were found residing within diffraction limited distance. When $\alpha 2 \beta$ GlyR was expressed with GFP fusion in only the $\beta$ subunit, bleaching steps ranged from 1 to 3 (Figure

135 , D-F). Since each $\alpha 2 \beta$ GlyR contains only $1 \beta$ subunit, 3 bleaching steps necessitates no less

14 than $3 \alpha 2 \beta$ GlyR within diffraction limited distance. Indeed, using a tri-binomial model with $p_{m}$

15 fixed at $62 \%$, we derived the fraction of spots containing 1, 2 and $3 \alpha 2 \beta$ GlyRs to be $36 \%, 33 \%$

16 and $31 \%$, respectively (Figure 5F light blue, see methods). As expected, our data cannot be

17 explained with only one $(\alpha 2)_{2} \beta_{3}$ GlyR being in each spot (Figure 5F, dashed lines).

\section{DISCUSSION}

19 Heteromeric GlyRs are the predominant form of GlyRs in adult animals. Research on embryonic/

20 early developmental forms of GlyRs, the homomeric GlyRs, provided very little information on

21 how adult heteromeric GlyRs work. Even the subunit stoichiometry was disputed for decades

22 (Burzomato et al., 2003; Durisic et al., 2012; Grudzinska et al., 2005; Kuhse et al., 1993; Patrizio

23 et al., 2017; Yang et al., 2012). In this work, we developed a system that allowed 
characterization of heteromeric GlyRs both in cells and in vitro. Our results showed unequivocally that each physiological $\alpha 2 \beta$ GlyR contains only one $\beta$ subunit and revealed the molecular mechanism underlying such arrangement. In addition, we identified structural features that gave rise to the specific electrophysiological properties, including glycine activation, singlechannel conductance and picrotoxin blocking, of heteromeric GlyRs and showed why GlyRs containing more than $1 \beta$ subunit do not conduct $\mathrm{Cl}^{-}$. We further found in cellular membranes that multiple GlyRs may exist within diffraction limited distance $(\sim 250 \mathrm{~nm})$. Through these findings, we pin-pointed the only physiological form of $\alpha 2 \beta$ GlyR that conducts $\mathrm{Cl}^{-}$, and revealed its working mechanism. GlyR clustering relies on a specific interaction between the $\beta$ subunit and the scaffolding protein gephyrin (Kirsch et al., 1993; Meyer et al., 1995), little can be learned from homomeric GlyRs since they do not contain the $\beta$ subunit. Our results have strong implications in GlyR clustering as follows (Figure 6). Since gephyrin assembles into a multimeric form with one GlyR $\beta$ subunit clustered - having only $1 \beta$ subunit requires the gephyrin to cluster by itself, GlyRs are recruited to the already formed gephyrin clusters. Having more than $1 \beta$ subunit in each GlyR would allow multi-valent interactions between the gephyrin and GlyR, inducing cluster formation. This is reminiscent of the phase-separated systems where some factors serve as scaffolds and others as clients (Banani et al., 2016) - the 4:1 $\alpha 2: \beta$ GlyR can only function as clients (Figure 6A) whereas additional $\beta$ subunits would allow GlyR to act as scaffolds of the inhibitory post-synaptic density (Figure 6B). Our data shows that functional GlyRs can only serve as clients, indicating that synaptic plasticity of a given glycinergic synapse may be regulated through two mechanisms: the 
1 physical properties of post-synaptic density, for examples size and compactness, can be regulated through gephyrin, while the intensity of glycinergic transmission can be further tuned through GlyRs diffusion in and out of clusters. Clearly, more research is required to gain complete

4 understanding.

Our results do not exclude the possibility of alternative subunit composition in native neuronal cells. First, we focused on the $\alpha 2 \beta$ GlyR, in contrast to $\alpha 1 \beta$ and/or $\alpha 3 \beta$ GlyR reported previously, raising the possibility that $4: 1$ is specific to the $\alpha 2 \beta$ GlyR. However, this seems unlikely because of the high sequence homology at the N-terminus and subunit interfaces (Figure S5 and S6).

9 Second, unidentified chaperons and other factors might produce GlyRs with multiple $\beta$ subunit in specific neuronal cells. In this case, since GlyRs containing multiple $\beta$ subunits are non-

11 conductive upon glycine-activation (Figure 4), these GlyRs will not function as ionotropic receptors, but instead as scaffolding proteins (Figure 6B). Multiple GlyRs residing within one diffraction-limited spot $(\sim 250 \mathrm{~nm}$ in diameter $)$ indicates that the number of $\beta$ subunits in each spot do not necessarily equal the number of $\beta$ subunits in each GlyR. Since we found this for both homomeric and heteromeric GlyRs, and in the absence of over-expressed gephyrin, the mechanism and physiological significance of this phenomenon calls for further investigations.

\section{ACKNOWLEDGMENTS}

We thank Robbie Boyed for preparing tissue culture, molecular biology and protein biochemistry tools for this project; Dr. Khuloud Jaqaman, Dr. Xian Cheng and Dr. Bruno Rocha Azevedo for

21 help in photobleaching data collection and analysis; Dr. Ryan Hibbs and Jeong Joo Kim for input

22 to biochemistry experiments and comments on the manuscript; Dr. Roderick MacKinnon, Dr.

23 Youxing Jiang and Dr. Xiao Tao for comments on the manuscript; All members of the Wang 
1 laboratory for helpful discussion. Cryo-EM data were collected at the University of Texas

2 Southwestern Medical Center Cryo-EM Facility, which is funded by the CPRIT Core Facility

3 Support Award RP170644. Funding: This work is supported by the Welch Foundation No. I-

$4 \quad 2020-20190330$.

6 AUTHOR CONTRIBUTIONS

7 H.Y. performed the experiments and analyzed the data. X.B. collected the Cryo-EM data and

8 reconstructed density maps. W.W. conceived the project, performed experiments, and analyzed

9 data. W.W., H.Y. and X.B. composed the manuscript.

11 DECLARATION OF INTERESTS

12 The authors declare no competing interests. 

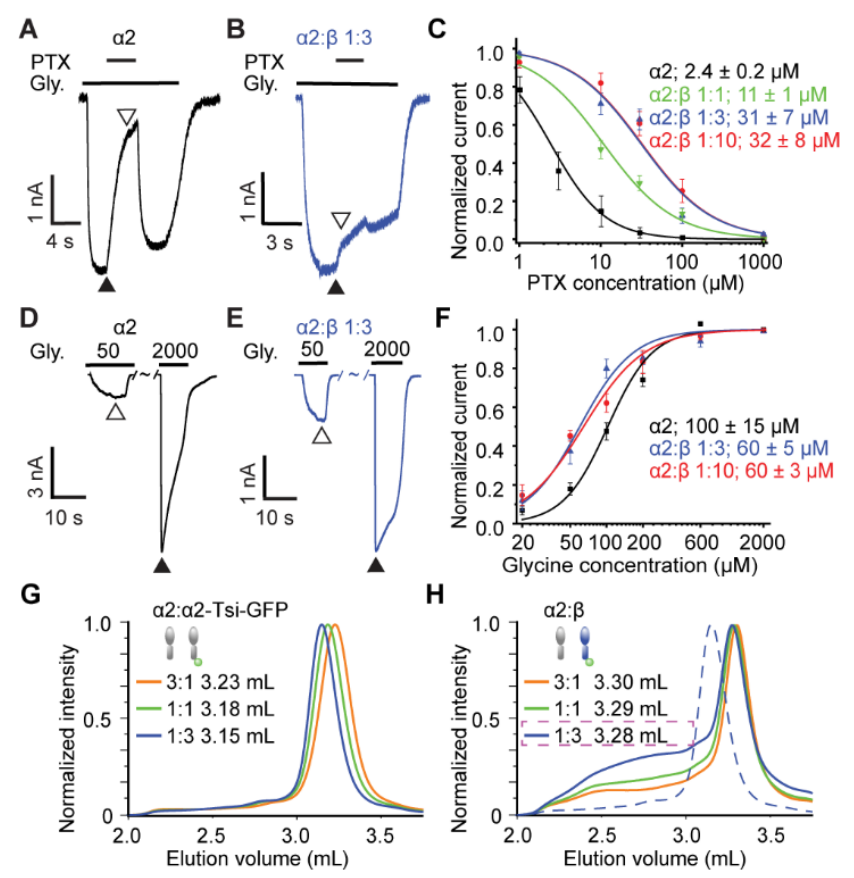

2

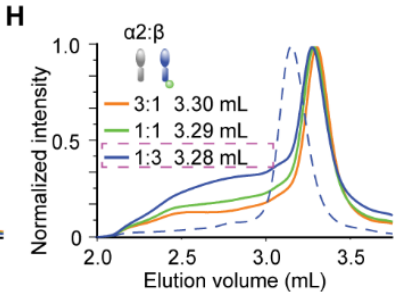

\section{Figure 1. Saturable effects of $\beta$ subunit expression levels}

4 (A and B) Representative recordings of GlyR inhibition by $10 \mu \mathrm{M}$ PTX in the presence of 100

$5 \mu \mathrm{M}$ glycine at $\alpha 2: \beta$ expression ratios of 1:0 (A) and 1:3 (B). Normalized current was calculated by dividing the currents immediately after (open triangle) PTX application by that before (closed

7 triangle).

8 (C) Dose response of PTX with IC50 resulting from Hill fits (lines) listed. Data are represented

$9 \quad$ as mean $\pm \operatorname{SEM}(\mathrm{n}=3-6$ cells $)$.

10 (D and E) Glycine activation at $\alpha 2: \beta$ ratios of 1:0 (D) and 1:3 (E). Glycine concentrations are in $11 \mu \mathrm{M}$.

12 (F) Dose response of glycine with EC50 resulting from Hill fits (lines) listed. Data are

13 represented as mean $\pm \operatorname{SEM}(n=3-6$ cells $)$. 
1 (G and H) FSEC elution profiles of $(\mathrm{G}) \alpha 2: \alpha 2-T$ si-GFP ratio of 3:1 (red), 1:1 (green) and 1:3

2 (blue) and (H) $\alpha 2: \beta$ ratio of 3:1 (red), 1:1 (green) and 1:3 (blue). Dashed line denotes 1:3 $\alpha 2: \alpha 2$ -

3 Tsi-GFP.Peak elution volumes are listed.

$4 \quad$ See also Figure S1. 

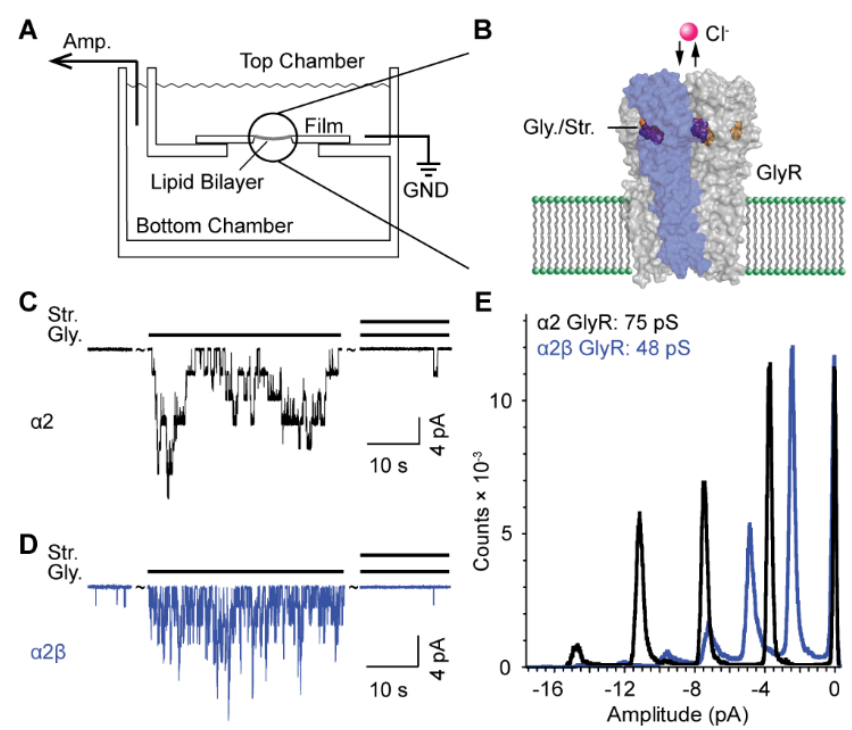

2 Figure. 2. Electrophysiology of GlyRs in planar lipid bilayers.

3 (A and B) Illustration of (A) the recording apparatus and (B) GlyR reconstituted into artificial

4 bilayers.

5 (C and D) Current traces of (C) $\alpha 2$ and (D) $\alpha 2 \beta$ GlyRs at $-50 \mathrm{mV}$ membrane potential. Solution

6 on both sides contained (in $\mathrm{mM}$ ): 10 potassium phosphate $\mathrm{pH} 7.4,150 \mathrm{KCl}$ and 1 EDTA.

7 Glycine (Gly.) and strychinine (Str.) concentrations were $200 \mu \mathrm{M}$ and $10 \mu \mathrm{M}$, respectively.

(E) Histogram of all points from traces of $\alpha 2$ homomer (color in black) and $\alpha 2 \beta$ heteromer (color

9 in blue). The conductance of $\alpha 2$ and $\alpha 2 \beta$ heteromer are listed. 
B

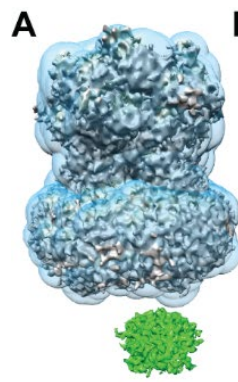

D

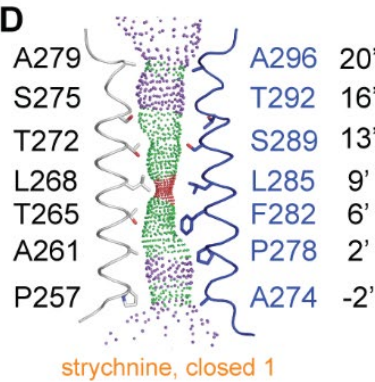

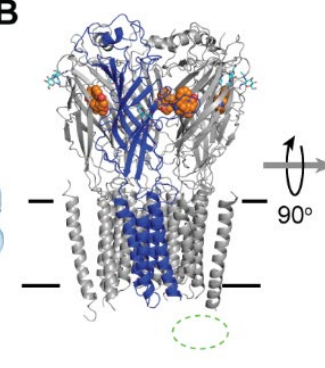

E

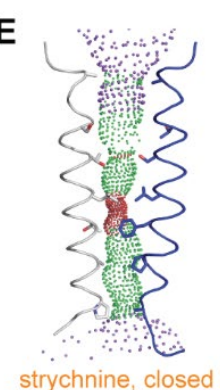

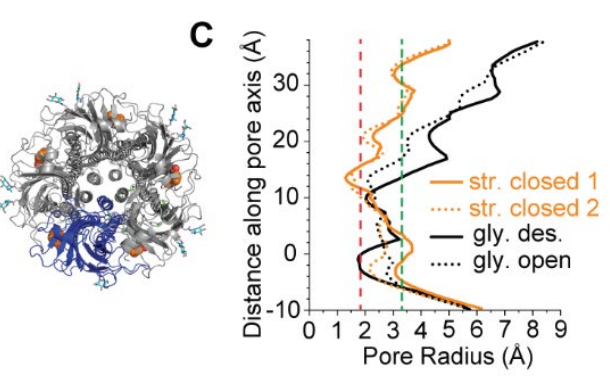

F

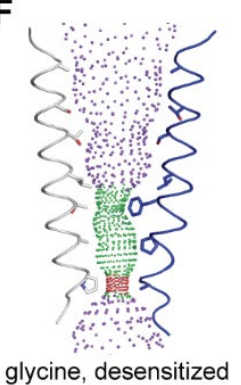

G

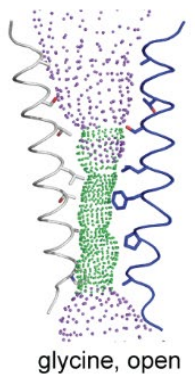

Figure 3. Overall structures of $\alpha 2 \beta$ GlyR in complex with glycine and strychnine.

(A) One $\alpha 2 \beta$ GlyR-strychnine cryo-EM density map containing GlyR (grey) and a GFP blob

(green), with a mask excluding GFP (translucent blue).

(B) Cartoon representations of $\alpha 2 \beta$ GlyR atomic model viewed parallel to the membrane (black

lines) plane (left) and down the extracellular side (right). Grey: $\alpha 2$, blue: $\beta$, orange: strychnine, cyan sticks: N-linked glycans.

(C) Pore radii plotted against the distance along the pore axis from the Ca position of 0 '

Arginine.

(D-G) Ion permeation pathways of (D) strychnine-bound closed 1, (E) closed 2, and (F) glycinebound desensitized, $(\mathrm{G})$ open states. Purple, green and red mesh represents radii of $>3.3 \AA$, 1.8 $3.3 \AA$ and $<1.8 \AA$, respectively. Sidechains of pore-lining residues are highlighted as sticks.

See also Figure S2, S3, S4 and S5. 
A

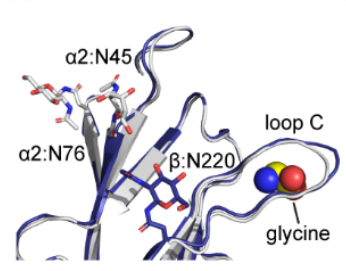

E

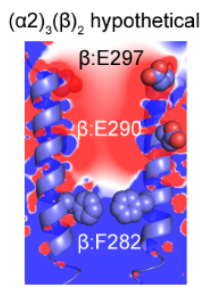

F
B
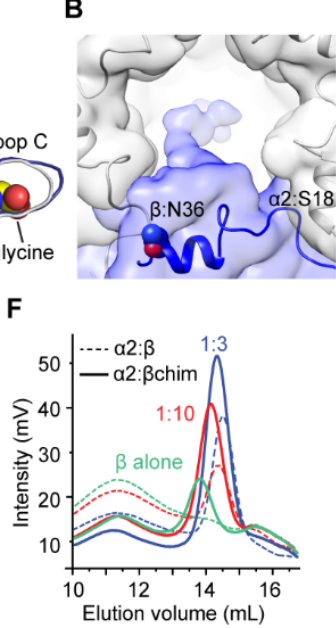

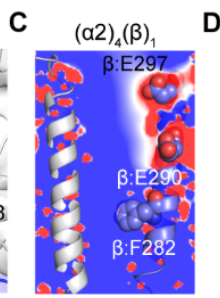

G

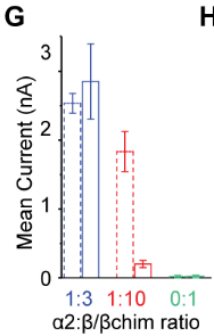

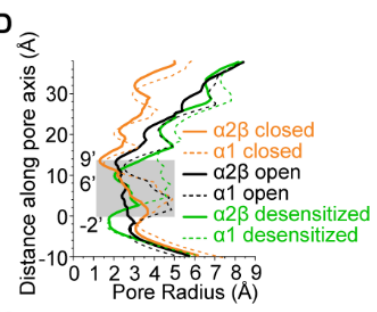

H

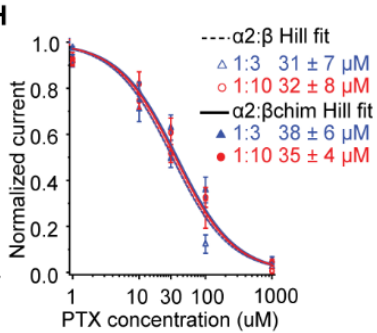

Figure 4. Features of the $\beta$ subunit underlying $\alpha 2 \beta$ GlyR physiology.

(A) N-linked glycosylation sites on $\alpha 2$ (grey) and $\beta$ (blue) subunits facing away the vestibule.

(B) cryo-EM density originating from $\beta$ :N36 (blue) and extending into the vestibule. Map is lowpass filtered and $6 \AA$ and shown as translucent surface.

(C) Ion permeation pore of $\alpha 2 \beta$ GlyR with $\beta$ : E297, E290 and F282 shown as spheres. Electrical potentials are shown as color gradient from red $(-2 \mathrm{kT} / \mathrm{e})$ to blue $(2 \mathrm{kT} / \mathrm{e})$.

(D) Pore radii in the closed, open and desensitized states of $\alpha 2 \beta$ (see Figure 3C) and homomeric al GlyRs (PDB ID: 3JAD, 6PM6, 6PM5, respectively). Regions exhibiting clear differences between $\alpha 2 \beta$ and $\alpha 1$ are shaded.

(E) Ion permeation pore of hypothetical $(\alpha 2) 3(\beta) 2$ represented as in $(C)$.

(F) FSEC of $\alpha 2$ co-expressed with $\beta$ (dashed) and $\beta$ chim (solid) at ratios of 1:3 (blue), 1:10 (red) and $\beta$ alone (light green).

(G) Whole-cell current activated by $100 \mu \mathrm{M}$ glycine $(\mathrm{n}=5-6$ cells, mean \pm SEM).

(H) PTX dose response curves of $\alpha 2: \beta$ and $\alpha 2: \beta$ chim expressed at 1:3 and 1:10 ratios $(\mathrm{n}=3-6$ cells, mean \pm SEM).

See also Figure S3, S5 and S6. 

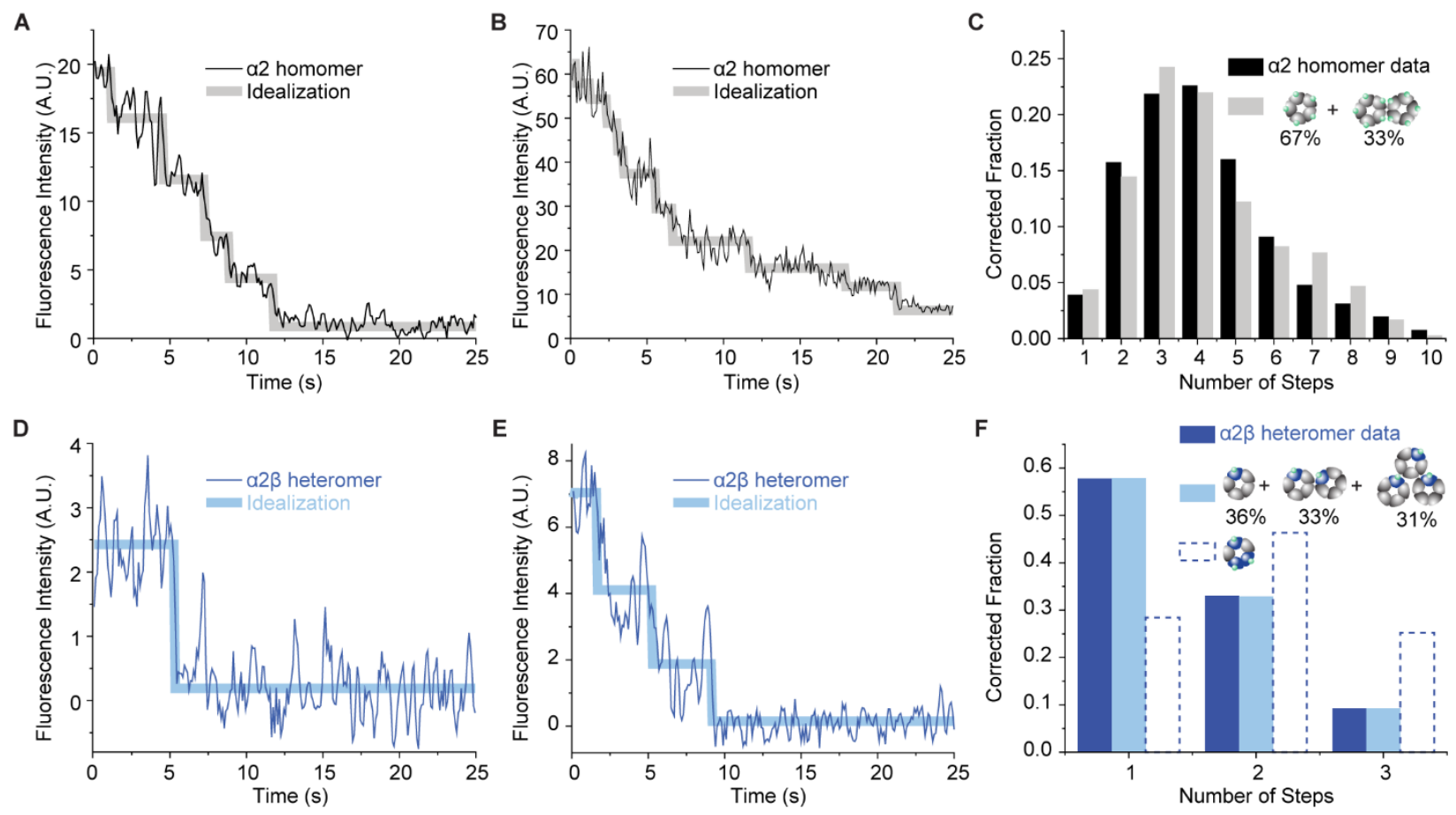

2 Figure 5. Step-wise photobleaching of GlyRs in HEK293 cells.

3 (A and B) Representative fluorescence intensities (black) and idealization (grey) of $\alpha 2$-GFP

4 exhibiting (A) 5 and (B) 10 photobleaching steps.

5 (C) Histogram of the $\alpha 2$-GFP GlyR steps from 2295 spots after correction for missed events

6 (black). A dual-binomial model was used to fit the data (grey), resulting in a GFP maturation rate

7 pm of $62 \%$.

9 and (E) 3 steps.

10

(F) Histogram of the $\alpha 2 \beta$ GlyR steps (blue) from 206 spots, shown with two binomial models

11 (cyan and dashed) using pm of 62\%. 
1
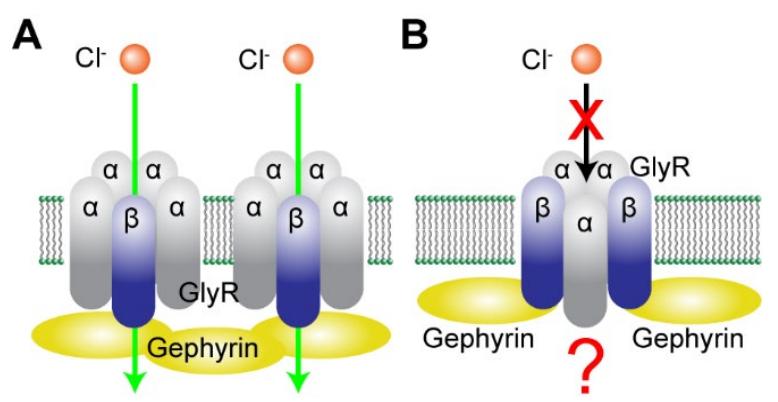

\section{Figure 6. Models of heteromeric GlyR - gephyrin clustering.}

(A) GlyRs containing only one $\beta$ subunit conducts $\mathrm{Cl}^{-}$upon activation and interact with alreadyformed gephyrin clusters in a monovalent manner.

(B) GlyRs containing two or more $\beta$ subunits may form multivalent interactions with gephyrin but do not conduct $\mathrm{Cl}^{-}$.

\section{METHODS}

\section{Cloning of hGlyR constructs}

DNAs coding human glycine receptor $\alpha 2$ (NCBI: NP_002054.1) and $\beta$ (NCBI: NP_000815.1) were amplified from cDNA clones (McDermott Center, UT Southwestern Medical center). The amino acid numbering starts from the first amino acid after signal peptide. For GlyR $\alpha 2$ subunit, region encoding M3/M4 loop (residues Q317-K381) was substituted by GSSG peptide, this construct was named as $\alpha 2 \mathrm{em}$. The $\beta \mathrm{em}$ construct contained the following modifications: region encoding M3/M4 loop (residues N334-N377) was removed and GGSSAAA-monomeric enhanced green fluorescent protein (mEGFP)(Zacharias et al., 2002) -SGSGSG was inserted. A

PA-tag (GVAMPGAEDDVV)(Fujii et al., 2014) and PreScission protease site

(LEVLFQ/GP)(Walker et al., 1994) were inserted after signal peptide at the N-terminus. For 
GlyR $\alpha 2-$ Tsi-GFP construct, region encoding M3/M4 loop (residues Q317-K381) was replaced with GGSSAAA-Tsi3(Li et al., 2013)-GSAGSAAGSG-mEGFP-SGSGSG. These mutants were cloned into a modified BacMam expression vector(Goehring et al., 2014). $\alpha 2$ wild type and a2em were cloned into pLVX-IRES-ZsGreen1 vector (Clonetech) for electrophysiology. The $\alpha 2 \mathrm{em}$ and $\beta \mathrm{em}$ constructs are used throughout this work and denoted $\alpha 2$ and $\beta$ for simplicity unless otherwise specified.

The BacMam vectors containing target protein were transformed into DH10BacY competent cells (Geneva Biotech) to produce bacmids, which in turn were used to produce viruses in $s f 9$

11 insect cells. The virus titer was determined using published methods(Goehring et al., 2014; Morales-Perez et al., 2016) and the virus ratios in this work denotes the multiplicity of infection (MOI) ratios. HEK293T adherent cells were infected with viruses at different $\alpha 2: \beta$ ratio or $\alpha 2: \alpha 2-T s i-G F P$ ratios $(1: 0,20: 1,10: 1,5: 1,3: 1,1: 1,1: 3,1: 5,1: 10,1: 20,0: 1$, total virus amount was kept constant). Expression was induced 20 hours after infection by adding $10 \mathrm{mM}$ sodium butyrate with $5 \mu \mathrm{M}$ strychnine added at the same time. Cells were collected 48 hours after induction by centrifugation at $20,000 \mathrm{~g}$ for 30 minutes at $4^{\circ} \mathrm{C}$. Cell pellets were resuspended and extracted with buffer A (40 mM Tris pH 8.0, $200 \mathrm{mM} \mathrm{NaCl}, 0.2 \mathrm{mM}$ PMSF, 0.75\%(w/v) n-

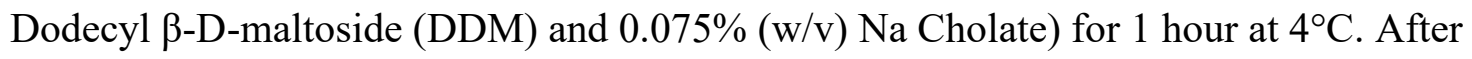
centrifuge, the supernatants were analyzed by FSEC on high-performance liquid chromatography system (HPLC, Shimadzu) equipped with fluorescence detectors set to read GFP fluorescence (excitation wavelength $480 \mathrm{~mm}$, emission wavelength $515 \mathrm{~mm}$ ). Due to differences in molecular 
1 weight ( $\alpha 245 \mathrm{KDa}, \beta$ 77KDa, $\alpha 2$-Tsi-GFP $87 \mathrm{KDa}$ ), elution volume was a function of subunit

2 stoichiometry.

$4 \quad$ Expression and purification of hGlyR

5 Large scale expression of hGlyR was essentially the same as in FSEC assays except for using

6 HEK293S GnTI- cells in suspension culture instead of adherent in FSEC. HEK293S GnTI- cells

7 were infected at a density of $2.5 \times 10^{6}$ cells $/ \mathrm{ml}$ using viruses encoding GlyR $\alpha 2$ and GlyR $\beta$ at

$8 \quad$ 1:3 ratio. Expression was induced 20 hours after infection with $10 \mathrm{mM}$ sodium butyrate and $5 \mu \mathrm{M}$

9 strychnine (or no strychnine for GlyR-glycine complex) added at the same time. 48 hours later,

10 cells were collected by centrifugation, then homogenized and extracted with buffer A for 1 hour

11 at $4^{\circ} \mathrm{C}$. PA-tag antibody (NZ-1)(Fujii et al., 2014) resin was used to capture hGlyR heteromer

12 from the extracted supernatant and then washed with buffer B (20 mM Tris pH 8.0, $200 \mathrm{mM}$

$13 \mathrm{NaCl}, 0.2 \mathrm{mM}$ PMSF, 0.1\% DDM, 0.02\% Na Cholate). PreScission protease was added to

14 release the hGlyR heteromer from PA-tag antibody resin. Eluent was concentrated and loaded

15 onto Superose 6 increase 10/300 GL size exclusion column (GE Healthcare) in buffer C (20 mM

16 Tris $\mathrm{pH} 8.0,200 \mathrm{mM} \mathrm{NaCl}, 0.025 \% \mathrm{DDM})$. Peak fractions were collected and concentrated to 4

$17 \mathrm{mg} / \mathrm{ml}$. GlyR $\alpha 2-$ Tsi-GFP homomer was prepared the same way except for that the Tse3(Li et

18 al., 2013) resin was used for purification. Buffer B containing $10 \mathrm{mM}$ EDTA was used to elute

19 hGlyR homomer from Tse3 resin. Concentrated proteins were used immediately.

$21 \quad$ Structure determination using single-particle cryo-EM

22 Reconstitution of $\alpha 2 \beta$ heteromer into saposin nanodisc 
1 Reconstitution of GlyR $\alpha 2 \beta$ heteromer into saposin nanodisc was modified from the published

2 protocol(Frauenfeld et al., 2016). After screening, a 1:30:200 molar ratio of $\alpha 2 \beta$ :saposin:brain

3 polar lipids extract (BPE) (Avanti) resulted in the best reconstitution with a small void peak

4 corresponding to lipid vesicles without scaffold. $100 \mu \mathrm{l} \alpha 2 \beta$ heteromer $(4.5 \mathrm{mg} / \mathrm{ml})$ and $17 \mu \mathrm{BPE}$

$5 \quad(13.6 \mathrm{mg} / \mathrm{ml}, 1 \% \mathrm{DDM})$ were mixed and put at room temperature (RT) for 10 minutes. Then

$6393 \mu \mathrm{l} \operatorname{saposin}(1.2 \mathrm{mg} / \mathrm{ml})$ was added and well mixed. The mixture was put at RT for 10 minutes,

7 then $3.2 \mathrm{~mL}$ dilution buffer (20mM Tris $\mathrm{pH} 8.0,200 \mathrm{mM} \mathrm{NaCl}, 1 \mathrm{mM}$ strychnine / 2mM glycine)

8 was added. After several minutes, bio-beads SM-2 (Bio-Rad) were added to the mixture and

9 rotated overnight at $4{ }^{\circ} \mathrm{C}$. Next morning, the mixture was put into a new tube to remove the bio-

10 beads, then fresh bio-beads were added again. After 8 hours, the mixture was centrifuged and

11 loaded onto Superose 6 increase size exclusion column in SEC buffer (20mM Tris pH 8.0,

$12 \quad 200 \mathrm{mM} \mathrm{NaCl}, 1 \mathrm{mM}$ strychnine / $2 \mathrm{mM}$ glycine). The peak fractions containing $\alpha 2 \beta$-saposin

13 nanodisc were concentrated to $4 \mathrm{mg} / \mathrm{mL}$ and used for EM grids preparation immediately.

14 Cryo-EM sample preparation, data collection and image processing

15 Fluorinated fos-choline 8 (Anatrace) was added into $4 \mathrm{mg} / \mathrm{ml} \alpha 2 \beta$ heteromer saposin nanodisc

16 sample at a final concentration of $1 \mathrm{cmc}$ just before freezing grids. $3.5 \mu 1$ sample applied to a

17 glow-discharged Quantifoil R1.2/1.3 400-mesh gold holey carbon grid (Quantifoil, Micro Tools

$18 \mathrm{GmbH}$, Germany), blotted under $100 \%$ humidity at $4{ }^{\circ} \mathrm{C}$ and plunged into liquid ethane using a

19 Mark IV Vitrobot (FEI). Micrographs were collected on a Titan Krios microscope (FEI) with a

$20 \mathrm{~K} 3$ Summit direct electron detector (Gatan) operating at $300 \mathrm{kV}$ using the SerialEM software.

21 The GIF-Quantum energy filter was set to a slit width of $20 \mathrm{eV}$. Images were recorded in the

22 super-resolution counting mode with the pixel size of $0.83 \AA$. Micrographs were dose-fractioned

23 into 32 (strychnine complex) or 50 (glycine complex) frames with a dose rate of $1.6 \mathrm{e}^{-} / \AA /$ frame. 
Motioncorr2 program(Zheng et al., 2017) was used to perform 2-fold binning, motion correction and dose weighting of the movie frames. CTF correction were carried out using the GCTF program(Zhang, 2016). The following image processing steps were carried out in RELION 3.0(Scheres, 2012). Auto-picked particles were extracted and binned by 4 times and used in 2D classification. Particles in good 2D classes were selected and subjected to 3D classification using an initial model downloaded from EMDB database (EMD-6346)(Du et al., 2015). 3D classification into 6 classes yielded 2 classes with good density for the entire channel. The cryoEM density of one GFP were identified in both classes, but present at different position with respect to the channel. As GFP is only fused to the $\beta$ subunit of the receptor, this 3D classification result suggests that there is only one $\beta$ subunit but four $\alpha$ subunits in the heteropentameric channel, and the relatively position of this single $\beta$ subunit with respect to the other four $\alpha$ subunits are different between these two good 3D classes. To put the $\beta$ subunit of both classes in the same position that would allow us to combine these two good classes, we rotated each particle from one class around the $\mathrm{Z}$ axis by $360^{\circ} / 5$ through manually modifying the column "_rlnAngleRot” in the RELION selection star file. As a result, a total of 111,378 particles for GlyR-strychnine and 268,380 particles for GlyR-glycine complexes from two good 3D classes were combined, re-extracted to the original pixel size. 3D refinement of these particles with $8^{\circ}$ local angular search and the $\mathrm{C} 1$ symmetry imposed led to a $3 \mathrm{D}$ reconstruction to overall resolutions of $3.8-3.9 \AA$, although the transmembrane region was resolved at relatively lower resolution. Therefore, the particles of the initial 3D reconstruction were subjected to partial signal subtraction to keep only the transmembrane region(Bai et al., 2015). 3D classification of these partial-subtracted particles without alignment led to two classes that showed much better density for the transmembrane domain (Figure S2A). After reverting to un-subtracted version, 
1 CTF refinement, particle polishing, and refinement using C1 symmetry, GlyR-strychnine complex yielded two classes. One class contained 38,541 particles and reached an overall resolution of $3.8 \AA$ (closed 1) and the other contained 44,965 particles and reached $3.6 \AA$ (closed 2). Closed 2 class exhibited a more 5-fold symmetrical TM arrangement (Figure S5). GlyRglycine complex also yielded two classes, one contained 55,816 particles with $3.8 \AA$ (open) resolution and the other contained 56,419 particles and reached $3.6 \AA$ (desensitized) resolution. Resolutions were estimated by applying a soft mask around the protein densities with the Fourier Shell Correlation (FCS) 0.143 criterion. Local resolutions were calculated using Resmap(Kucukelbir et al., 2014).

\section{Model building and refinement}

Model building of GlyR $\alpha 2 \beta$ heteromer was initiated by docking the structure of $\alpha 1$ homomer strychnine-bound state(Du et al., 2015) (PDB ID: 3JAD) into the density map of GlyR $\alpha 2 \beta$ heteromer using Chimera(Pettersen et al., 2004). Amino acids were mutated according to $\alpha 2$ and $\beta$ sequences and rebuilt in $\operatorname{Coot}($ Emsley et al., 2010). The structure model was manually adjusted in Coot, then refinement was performed with real-space refinement module with secondary structure restraints in PHENIX package(Adams et al., 2010; Liebschner et al., 2019). MolProbity was used to validate the geometries of the refined models(Williams et al., 2018). Fourier shell correlation (FSC) curves were calculated between refined atomic model and the work/free half maps as well as the full map to assess the correlation between the model and density map. Statistics of cryo-EM data processing and model refinement are listed in Table S1. Pore radii were calculated using the HOLE program(Smart et al., 1996). Figures were prepared in Chimera or PyMOL(Schrödinger, 2015). Electrostatics were calculated using Adaptive PoissonBoltzmann plugin at $37^{\circ} \mathrm{C}$ and with $150 \mathrm{mM}$ of monovalent anion/cations (Baker et al., 2001). 
1 The final model of strychnine closed 2 contained the $\alpha 2$ and $\beta$ subunit amino acids except the

2 following: $\alpha 2$ subunit (total 364aa, 339aa built, 25aa not built) K1-T14, GSSG linker, K382 and

3 E420- K425; $\beta$ subunit (total 444aa, 338aa built, 106aa not built) K1-Q20, L332-N333,

4 GGSSAAA-EGFP-SGSGSG insertion and V378-A448. Q21-R28 of $\beta$ subunit were modeled as

5 poly-alanine due to limit in resolution. The model for strychnine closed 1 is the same as closed 2

6 except for Q21-R28 of $\beta$ subunit were not modeled. The final model of glycine bound

7 desensitized state contained the $\alpha 2$ and $\beta$ subunit amino acids except the following: $\alpha 2$ subunit

8 (total 364aa, 338aa built, 26aa not built) K1-T14, GSSG linker, K382 and H419- K425; $\beta$

9 subunit (total 444aa, 337aa built, 107aa not built) K1-R28, GGSSAAA-EGFP-SGSGSG

10 insertion and V378-V443. The model for glycine bound open state is the same as desensitized

11 state except that only 3 glycine are modeled.

13 Electrophysiology

$14 \quad$ Whole cell patch clamp

15 Plasmids bearing GlyR constructs were transiently transfected into HEK293T cells by

16 Lipofectamine 3000 (Invitrogen) following manufacturer's protocol. For each $35 \mathrm{~mm}$ dish, 1.5

$17 \mu \mathrm{g}$ of DNA was transfected at different $\alpha: \beta$ ratios $(1: 0,1: 1,1: 3,1: 10)$. After transfection, cells

18 were cultured at $37^{\circ} \mathrm{C}$ for $6 \sim 8$ hours, then transferred to $30^{\circ} \mathrm{C}$ cultured for $\sim 12$ hours. Whole-cell

19 recordings were performed at room-temperature. The bath solution contained (in $\mathrm{mM}$ ): 10

20 HEPES pH 7.4, $10 \mathrm{KCl}, 125 \mathrm{NaCl}, 2 \mathrm{MgCl}_{2}, 1 \mathrm{CaCl}_{2}$ and 10 glucose. The pipette solution

21 contained (in $\mathrm{mM}$ ): $10 \mathrm{HEPES} \mathrm{pH} 7.4,150 \mathrm{KCl}, 5 \mathrm{NaCl}, 2 \mathrm{MgCl}_{2}, 1 \mathrm{CaCl}_{2}$ and 5 EGTA.

22 Borosilicate glass pipettes with resistance between 2 7 M $\Omega$ were used. A Digidata 1550B

23 digitizer (Molecular Devices) was connected to an Axopatch 200B amplifier (Molecular 
Devices) for data acquisition. Analog signals were filtered at $1 \mathrm{kHz}$ and subsequently sampled at $20 \mathrm{kHz}$ and stored on a computer running pClamp 10.5 software. Membrane was held at $-80 \mathrm{mV}$ during perfusion of ligands to record GlyR current. For the heteromer recordings, as the GlyR $\beta$ construct had an EGFP insertion, we used GFP fluorescence to identify the cells expressing the GlyR $\beta$ subunit. Hill equation was used to fit the dose-response data and derive the $\mathrm{EC}_{50}(k)$ and Hill coefficient $(n)$. For glycine activation, we used $I=I_{0}+\left(I_{\max }-I_{0}\right) \frac{x^{n}}{k^{n}+x^{n}}$, where $I$ is current, $I_{0}$ is the basal current (spontaneous opening current and leak, very close to 0 ), $I_{\max }$ is the maximum current and $x$ is glycine concentration. Hill equation was also used to fit picrotoxin inhibition data: $I=I_{\max }-\left(I_{\max }-I_{0}\right) \frac{x^{n}}{k^{n}+x^{n}}$, with same definitions of symbols except that $k$ represents $\mathrm{IC}_{50}$. Data was fitted with software OriginPro (OriginLab, Northampton, MA). Measurements were from 3-6 cells, average and S.E.M. values were calculated for each data point.

\section{Proteoliposome reconstitution}

Purified $\alpha 2 \beta$ heteromer and $\alpha 2$ homomer were reconstituted into proteoliposomes using the lipid mixture composed of 3:1 (wt:wt) 1-palmitoyl-2-oleoyl-sn-glycero-3-phosphoethanolamine (POPE) : 1-palmitoyl-2-oleoyl-sn-glycero-3-phospho-1'-rac-glycerol) (POPG). The reconstitution procedure is similar to that previously reported(Wang et al., 2014). Briefly, 20 $\mathrm{mg} / \mathrm{ml}$ of the above lipid mixture was dispersed by sonication until translucent and then solubilized with $1 \%$ DDM. GlyR was diluted with reconstitution buffer $(10 \mathrm{mM}$ potassium phosphate $\mathrm{pH}$ 7.4, $150 \mathrm{mM} \mathrm{KCl}, 1 \mathrm{mM}$ EDTA and $3 \mathrm{mM}$ DTT) supplemented with $0.2 \%$ DDM to $2 \mathrm{mg} / \mathrm{ml}$. Equal volumes of $20 \mathrm{mg} / \mathrm{ml}$ solubilized lipid mixture was combined with the diluted GlyR solutions, resulting in a final protein:lipid (wt:wt) ratios of 1:10 at a lipid concentration of $\sim 10 \mathrm{mg} / \mathrm{ml}$. Detergent was removed by dialysis against reconstitution buffer for 4 times, 12 
1 hours each at $4^{\circ} \mathrm{C}$. Bio-Beads SM2 (Bio-Rad) were added outside the dialysis bag $(\sim 1 \mathrm{~g} / 500 \mathrm{~mL}$

2 buffer) in the last three cycles. $20 \mu \mathrm{L}$ aliquots of the proteoliposomes were frozen stored at -80

$3{ }^{\circ} \mathrm{C}$ until needed.

$4 \quad$ Planar lipid bilayer recording

5 The planer bilayer recording experiments are similar to previously described(Wang et al., 2016;

6 Wang et al., 2014). Briefly, $20 \mathrm{mg} / \mathrm{ml}$ of lipid mixture composed of 2:1:1(wt:wt:wt) of

7 DOPE:POPC:POPS, or brain polar extract (Avanti ), was dissolved in decane and used to form

8 planar lipid bilayer over a $\sim 100 \mu \mathrm{m}$ hole on a piece of transparency film using the painting

9 method. The same solution (10 mM potassium phosphate $\mathrm{pH}$ 7.4, $150 \mathrm{mM} \mathrm{KCl,} 1 \mathrm{mM}$ EDTA)

10 was used in both chambers. Proteoliposomes were mixed with $1 \mathrm{M} \mathrm{KCl}$ at equal volume and

11 sonicated to facilitate vesicle fusion into planer bilayer immediately before use. After vesicle

12 fusion into planer bilayer, buffer containing glycine or strychnine was applied on the planar

13 bilayer manually. A Digidata 1550B digitizer (Molecular Devices) interfaced to pClamp10.5

14 software was connected to an Axopatch 200B amplifier (Molecular Devices) for data acquisition.

15 Analog signals were filtered at $1 \mathrm{kHz}$ and subsequently sampled at $20 \mathrm{kHz}$. Recordings were

16 performed at room temperature. Membrane voltage was held at $-50 \mathrm{mV}$. The concentrations of

17 glycine and strychnine applied to the membrane were $200 \mu \mathrm{M}$ and $10 \mu \mathrm{M}$, respectively.

19 Step-wise Photobleaching

$20 \alpha 2-G F P$ or $\alpha 2: \beta$ at 1:3 ratio was transfected into HEK293T cells on collagen coated glass bottom

21 dishes using lipofectamine 3000 (Invitrogen) for expression of $\alpha 2$ GlyR or $\alpha 2 \beta$ GlyR,

22 respectively. The transfected cells were kept in $\mathrm{CO}_{2}$ incubator for 6 hours at $37^{\circ} \mathrm{C}$ and then

23 removed to $30^{\circ} \mathrm{C}$ for overnight expression. Cells were unroofed(Usukura et al., 2016) and fixed 
1 prior to imaging as follows: After 3 times wash at room temperature (RT) with PhosphateBuffered Saline PBS (10 mM Na $2 \mathrm{HPO}_{4}, 1.8 \mathrm{mM} \mathrm{NaH}_{2} \mathrm{PO}_{4}, 137 \mathrm{mM} \mathrm{NaCl}, 3 \mathrm{mM} \mathrm{KCl}$ ), cells were sonicated at 20\% power for $1 \mathrm{~s}$ using a Branson SFX550 sonicator equipped with a flat microtip in ice-cold PBS supplemented with $\%$ para-formaldehyde (PFA), $2 \mathrm{mM} \mathrm{MgCl} 2$ and 1 $\mathrm{mM} \mathrm{CaCl}_{2}$. Unroofed cells were washed quickly with ice-cold PBS and immediately switched into PBS containing 4\% PFA at RT and fixed for $15 \mathrm{~min}$. After 3 times washing in PBS for 10 min each, the cells were imaged within 2 hours. Total internal reflection illumination was achieved using an in-house built prism-type system using the Gem 488 laser (Laser Quantum) and components from Thorlabs at 10\% power. A 1.5 OD neutral density filter was used during searching of cells to minimize photobleaching. A Leica DM6 FS microscope equipped with a 63x 1.2 NA CS2 water immersion objective and a Hamamatsu flash 4.0 V3 camera was used for imaging. Movies were collected using a PC running Metamorph (Molecular Devices) with 100 cropped using ImageJ(Schneider et al., 2012) and analyzed using the PIF program(McGuire et al., 2012) to generate statistics of photobleaching steps. A dual-binomial model formulated allowing one and two pentameric GlyRs in each diffraction-limited fluorescence spot was used to fit the photobleaching step distribution of homomeric $\alpha 2 \mathrm{G}$ GlyR as follows:

8

$$
\begin{gathered}
\left.s(n)\right|_{1 \leq n \leq 10}=\frac{\left.(1-f) \frac{5 !}{(5-n) ! n !} p_{m}{ }^{n}\left(1-p_{m}\right)^{5-n}\right|_{n \leq 5}+f \frac{10 !}{(10-n) ! n !} p_{m}{ }^{n}\left(1-p_{m}\right)^{10-n}}{1-s_{0}} \\
s_{0}=(1-f) \frac{5 !}{(5-0) ! 0 !} p_{m}{ }^{0}\left(1-p_{m}\right)^{5-0}+f \frac{10 !}{(10-0) ! 0 !} p_{m}{ }^{0}\left(1-p_{m}\right)^{10-0}
\end{gathered}
$$

Where $s(n)$ is the fraction of spots with $n$ photobleaching steps corrected for the fraction of non-fluorescent events $s_{0} . p_{m}$ and $f$ were the fitted parameters, which denote the maturation rate of GFP and the fraction of spots with two $\alpha 2$ GlyRs, respectively. Similarly, a tri-binomial model 
1 assuming simultaneous presence of 1, 2 and 3 GlyRs in each spot was used for the heteromeric $\alpha 2 \beta$ GlyR photobleaching steps as follows

$$
\left.s(n)\right|_{1 \leq n \leq 3}
$$

4

$$
\begin{gathered}
=\frac{\left.\left(1-f_{1}-f_{2}\right) p_{m}^{n}\left(1-p_{m}\right)^{1-n}\right|_{n \leq 1}+\left.f_{1} \frac{2 !}{(2-n) ! n !} p_{m}^{n}\left(1-p_{m}\right)^{2-n}\right|_{n \leq 2}+f_{2} \frac{3 !}{(3-n) ! n !} p_{m}^{n}\left(1-p_{m}\right)^{3-n}}{1-s_{0}} \\
s_{0}=\left(1-f_{1}-f_{2}\right)\left(1-p_{m}+f_{1} \frac{2 !}{(2-0) ! 0 !} p_{m}{ }^{0}\left(1-p_{m}\right)^{2-0}+f_{2} \frac{3 !}{(3-0) ! 0 !} p_{m}{ }^{0}\left(1-p_{m}\right)^{3-0}\right.
\end{gathered}
$$

Where $f_{1}$ and $f_{2}$ were the fitted parameters which denote the corrected fractions of spots with 2 and $3 \alpha 2 \beta$ GlyRs, respectively. $p_{m}$ was fixed at 0.62 (the value from $\alpha 2$ GlyR). Traces and statistics were plotted using OriginPro (OriginLab).

\section{$\underline{\text { Western Blotting }}$}

For GlyR $\alpha 2$ subunit, a V5-tag (KEGKPIPNPLLGLDSTAA) was inserted into the $\alpha 2 \mathrm{em}$ construct after signal peptide at the N-terminus. Plasmids bearing GlyR constructs were transiently transfected into HEK293T cells by Lipofectamine 3000 (Invitrogen) following manufacturer's protocol at different $\alpha 2: \beta$ DNA ratios $(1: 0,3: 1,1: 1,1: 3,1: 10,0: 1)$. After transfection, cells were cultured at $37^{\circ} \mathrm{C}$ for 20 hours, then transferred to $30^{\circ} \mathrm{C}$ for additional $\sim 8$ hours. After detergent extraction, supernatants were mixed with an equal volume of loading buffer containing 4\% SDS and 10\% DTT. Then samples were analyzed using SDS-PAGE with Bio-Rad 4-15\% TGX gels and transferred onto nitrocellulose membrane. Western Blot was performed with anti-V5-tag (Cell Signaling Technology, D3H8Q) for $\alpha 2$ or Anti-GFP (Santa Cruz Biotechnology, sc-9996) for $\beta$. 
Data Resources

3 Atomic coordinates of $\alpha 2 \beta$ glycine receptor have been deposited in the Protein Data Bank with

4 accession numbers 7KUY for GlyR $\alpha 2 \beta$ closed state 2, 7L31 for GlyR closed state 1, 5BKG for

5 glycine bound open state and 5BKF for glycine bound desensitized state. The corresponding

6 cryo-EM density maps have been deposited with the Electron Microscopy Data Bank using

7 accession numbers EMD-23041 for $\alpha 2 \beta$ closed state 2 and EMD- 23148 for $\alpha 2 \beta$ closed state 1 ,

8 EMD-9404 for glycine bound open state and EMD-9403 for glycine bound desensitized state. 


\section{REFERENCES}

Adams, P.D., Afonine, P.V., Bunkoczi, G., Chen, V.B., Davis, I.W., Echols, N., Headd, J.J., Hung, L.W., Kapral, G.J., Grosse-Kunstleve, R.W., et al. (2010). PHENIX: a comprehensive Python-based system for macromolecular structure solution. Acta Crystallogr D Biol Crystallogr $66,213-221$.

Ali, D.W., Drapeau, P., and Legendre, P. (2000). Development of spontaneous glycinergic currents in the Mauthner neuron of the zebrafish embryo. Journal of neurophysiology 84,1726 1736.

Bai, X.C., Rajendra, E., Yang, G., Shi, Y., and Scheres, S.H. (2015). Sampling the conformational space of the catalytic subunit of human gamma-secretase. Elife 4.

Baker, N.A., Sept, D., Joseph, S., Holst, M.J., and McCammon, J.A. (2001). Electrostatics of nanosystems: application to microtubules and the ribosome. Proceedings of the National Academy of Sciences of the United States of America 98, 10037-10041.

Banani, S.F., Rice, A.M., Peeples, W.B., Lin, Y., Jain, S., Parker, R., and Rosen, M.K. (2016). Compositional Control of Phase-Separated Cellular Bodies. Cell 166, 651-663.

Becker, C.M., Hoch, W., and Betz, H. (1988). Glycine receptor heterogeneity in rat spinal cord during postnatal development. The EMBO journal 7, 3717-3726.

Bode, A., and Lynch, J.W. (2014). The impact of human hyperekplexia mutations on glycine receptor structure and function. Molecular brain 7, 2.

Bormann, J., Rundstrom, N., Betz, H., and Langosch, D. (1993). Residues within transmembrane segment M2 determine chloride conductance of glycine receptor homo- and hetero-oligomers. The EMBO journal 12, 3729-3737. 
1 Burzomato, V., Groot-Kormelink, P.J., Sivilotti, L.G., and Beato, M. (2003). Stoichiometry of

2 recombinant heteromeric glycine receptors revealed by a pore-lining region point mutation.

3 Receptors Channels 9, 353-361.

4 Cascio, M., Schoppa, N.E., Grodzicki, R.L., Sigworth, F.J., and Fox, R.O. (1993). Functional

5 expression and purification of a homomeric human alpha 1 glycine receptor in baculovirus-

6 infected insect cells. The Journal of biological chemistry 268, 22135-22142.

7 Cascio, M., Shenkel, S., Grodzicki, R.L., Sigworth, F.J., and Fox, R.O. (2001). Functional

8 reconstitution and characterization of recombinant human alpha 1-glycine receptors. The Journal

9 of biological chemistry 276, 20981-20988.

10 Citri, A., and Malenka, R.C. (2008). Synaptic plasticity: multiple forms, functions, and

11 mechanisms. Neuropsychopharmacology : official publication of the American College of

12 Neuropsychopharmacology 33, 18-41.

13 Du, J., Lu, W., Wu, S., Cheng, Y., and Gouaux, E. (2015). Glycine receptor mechanism

14 elucidated by electron cryo-microscopy. Nature 526, 224-229.

15 Durisic, N., Godin, A.G., Wever, C.M., Heyes, C.D., Lakadamyali, M., and Dent, J.A. (2012).

16 Stoichiometry of the human glycine receptor revealed by direct subunit counting. The Journal of

17 neuroscience : the official journal of the Society for Neuroscience 32, 12915-12920.

18 Eccles, J.C. (1982). The synapse: from electrical to chemical transmission. Annual review of

19 neuroscience 5, 325-339.

20 Emsley, P., Lohkamp, B., Scott, W.G., and Cowtan, K. (2010). Features and development of

21 Coot. Acta Crystallogr D Biol Crystallogr 66, 486-501. 
1 Flayhan, A., Mertens, H.D.T., Ural-Blimke, Y., Martinez Molledo, M., Svergun, D.I., and Low,

2 C. (2018). Saposin Lipid Nanoparticles: A Highly Versatile and Modular Tool for Membrane

3 Protein Research. Structure 26, 345-355 e345.

4 Frauenfeld, J., Loving, R., Armache, J.P., Sonnen, A.F., Guettou, F., Moberg, P., Zhu, L.,

5 Jegerschold, C., Flayhan, A., Briggs, J.A., et al. (2016). A saposin-lipoprotein nanoparticle

6 system for membrane proteins. Nat Methods 13, 345-351.

7 Fujii, Y., Kaneko, M., Neyazaki, M., Nogi, T., Kato, Y., and Takagi, J. (2014). PA tag: a

8 versatile protein tagging system using a super high affinity antibody against a dodecapeptide

9 derived from human podoplanin. Protein Expr Purif 95, 240-247.

10 Goehring, A., Lee, C.H., Wang, K.H., Michel, J.C., Claxton, D.P., Baconguis, I., Althoff, T.,

11 Fischer, S., Garcia, K.C., and Gouaux, E. (2014). Screening and large-scale expression of

12 membrane proteins in mammalian cells for structural studies. Nat Protoc 9, 2574-2585.

13 Griffon, N., Buttner, C., Nicke, A., Kuhse, J., Schmalzing, G., and Betz, H. (1999). Molecular

14 determinants of glycine receptor subunit assembly. The EMBO journal 18, 4711-4721.

15 Grudzinska, J., Schemm, R., Haeger, S., Nicke, A., Schmalzing, G., Betz, H., and Laube, B.

16 (2005). The beta subunit determines the ligand binding properties of synaptic glycine receptors.

$17 \quad$ Neuron 45, 727-739.

18 Harvey, R.J., Depner, U.B., Wassle, H., Ahmadi, S., Heindl, C., Reinold, H., Smart, T.G.,

19 Harvey, K., Schutz, B., Abo-Salem, O.M., et al. (2004). GlyR alpha3: an essential target for

20 spinal PGE2-mediated inflammatory pain sensitization. Science 304, 884-887.

21 Huang, X., Chen, H., Michelsen, K., Schneider, S., and Shaffer, P.L. (2015). Crystal structure of

22 human glycine receptor-alpha3 bound to antagonist strychnine. Nature 526, 277-280. 
1 Kirsch, J., Wolters, I., Triller, A., and Betz, H. (1993). Gephyrin antisense oligonucleotides

2 prevent glycine receptor clustering in spinal neurons. Nature 366, 745-748.

3 Kucukelbir, A., Sigworth, F.J., and Tagare, H.D. (2014). Quantifying the local resolution of

4 cryo-EM density maps. Nat Methods 11, 63-65.

5 Kuhse, J., Laube, B., Magalei, D., and Betz, H. (1993). Assembly of the inhibitory glycine

6 receptor: identification of amino acid sequence motifs governing subunit stoichiometry. Neuron

$7 \quad 11,1049-1056$.

8 Kumar, A., Basak, S., Rao, S., Gicheru, Y., Mayer, M.L., Sansom, M.S.P., and Chakrapani, S.

9 (2020). Mechanisms of activation and desensitization of full-length glycine receptor in lipid

10 nanodiscs. Nature communications $11,3752$.

11 Langosch, D., Thomas, L., and Betz, H. (1988). Conserved quaternary structure of ligand-gated

12 ion channels: the postsynaptic glycine receptor is a pentamer. Proceedings of the National

13 Academy of Sciences of the United States of America 85, 7394-7398.

14 Li, L., Zhang, W., Liu, Q., Gao, Y., Gao, Y., Wang, Y., Wang, D.Z., Li, Z., and Wang, T.

15 (2013). Structural Insights on the bacteriolytic and self-protection mechanism of muramidase

16 effector Tse3 in Pseudomonas aeruginosa. J Biol Chem 288, 30607-30613.

17 Liebschner, D., Afonine, P.V., Baker, M.L., Bunkoczi, G., Chen, V.B., Croll, T.I., Hintze, B.,

18 Hung, L.W., Jain, S., McCoy, A.J., et al. (2019). Macromolecular structure determination using

19 X-rays, neutrons and electrons: recent developments in Phenix. Acta Crystallogr D Struct Biol

$20 \quad 75,861-877$.

21 Lynch, J.W. (2004). Molecular structure and function of the glycine receptor chloride channel.

22 Physiological reviews 84, 1051-1095. 
1 Lynch, J.W. (2009). Native glycine receptor subtypes and their physiological roles.

2 Neuropharmacology 56, 303-309.

3 Lynch, J.W., and Callister, R.J. (2006). Glycine receptors: a new therapeutic target in pain

4 pathways. Current opinion in investigational drugs 7, 48-53.

5 Malosio, M.L., Marqueze-Pouey, B., Kuhse, J., and Betz, H. (1991). Widespread expression of

6 glycine receptor subunit mRNAs in the adult and developing rat brain. The EMBO journal 10,

7 2401-2409.

8 McGuire, H., Aurousseau, M.R., Bowie, D., and Blunck, R. (2012). Automating single subunit

9 counting of membrane proteins in mammalian cells. The Journal of biological chemistry 287 ,

$10 \quad 35912-35921$.

11 Meyer, G., Kirsch, J., Betz, H., and Langosch, D. (1995). Identification of a gephyrin binding

12 motif on the glycine receptor beta subunit. Neuron 15, 563-572.

13 Mohammadi, B., Krampfl, K., Cetinkaya, C., Moschref, H., Grosskreutz, J., Dengler, R., and

14 Bufler, J. (2003). Kinetic analysis of recombinant mammalian alpha(1) and alpha(1)beta glycine

15 receptor channels. Eur Biophys J 32, 529-536.

16 Morales-Perez, C.L., Noviello, C.M., and Hibbs, R.E. (2016). Manipulation of Subunit

17 Stoichiometry in Heteromeric Membrane Proteins. Structure 24, 797-805.

18 Moss, S.J., and Smart, T.G. (2001). Constructing inhibitory synapses. Nature reviews

19 Neuroscience 2, 240-250.

20 Mueller, P., Rudin, D.O., Tien, H.T., and Wescott, W.C. (1962). Reconstitution of cell

21 membrane structure in vitro and its transformation into an excitable system. Nature 194, 979-

22980. 
1 Patrizio, A., Renner, M., Pizzarelli, R., Triller, A., and Specht, C.G. (2017). Alpha subunitdependent glycine receptor clustering and regulation of synaptic receptor numbers. Scientific reports $7,10899$.

Patrizio, A., and Specht, C.G. (2016). Counting numbers of synaptic proteins: absolute quantification and single molecule imaging techniques. Neurophotonics 3, 041805. Pettersen, E.F., Goddard, T.D., Huang, C.C., Couch, G.S., Greenblatt, D.M., Meng, E.C., and analysis. J Comput Chem 25, 1605-1612.

9 Pfeiffer, F., and Betz, H. (1981). Solubilization of the glycine receptor from rat spinal cord. Brain Res 226, 273-279.

11 Phulera, S., Zhu, H., Yu, J., Claxton, D.P., Yoder, N., Yoshioka, C., and Gouaux, E. (2018).

12 Cryo-EM structure of the benzodiazepine-sensitive alpha1beta1gamma2S tri-heteromeric

13 GABAA receptor in complex with GABA. eLife 7.

14 Pribilla, I., Takagi, T., Langosch, D., Bormann, J., and Betz, H. (1992). The atypical M2 segment

15 of the beta subunit confers picrotoxinin resistance to inhibitory glycine receptor channels. The EMBO journal 11, 4305-4311.

17 Rahman, M.M., Teng, J., Worrell, B.T., Noviello, C.M., Lee, M., Karlin, A., Stowell, M.H.B., 18 and Hibbs, R.E. (2020). Structure of the Native Muscle-type Nicotinic Receptor and Inhibition 19 by Snake Venom Toxins. Neuron 106, 952-962 e955.

20 Sander, B., Tria, G., Shkumatov, A.V., Kim, E.Y., Grossmann, J.G., Tessmer, I., Svergun, D.I., 21 and Schindelin, H. (2013). Structural characterization of gephyrin by AFM and SAXS reveals a 22 mixture of compact and extended states. Acta crystallographica Section D, Biological crystallography $69,2050-2060$. 
1 Schaefer, N., Roemer, V., Janzen, D., and Villmann, C. (2018). Impaired Glycine Receptor

2 Trafficking in Neurological Diseases. Frontiers in molecular neuroscience 11, 291.

3 Scheres, S.H.W. (2012). RELION: Implementation of a Bayesian approach to cryo-EM structure

4 determination. J Struct Biol 180, 519-530.

5 Schmidt, T., Schutz, G.J., Gruber, H.J., and Schindler, H. (1996). Local stoichiometries

6 determined by counting individual molecules. Analytical chemistry 68, 4397-4401.

7 Schmieden, V., Grenningloh, G., Schofield, P.R., and Betz, H. (1989). Functional expression in

8 Xenopus oocytes of the strychnine binding $48 \mathrm{kd}$ subunit of the glycine receptor. The EMBO

9 journal $8,695-700$.

10 Schneider, C.A., Rasband, W.S., and Eliceiri, K.W. (2012). NIH Image to ImageJ: 25 years of

11 image analysis. Nat Methods 9, 671-675.

12 Schrödinger, L. (2015). The PyMOL molecular graphics system, version 2.3.1.

13 Shan, Q., Haddrill, J.L., and Lynch, J.W. (2001). A single beta subunit M2 domain residue

14 controls the picrotoxin sensitivity of alphabeta heteromeric glycine receptor chloride channels. J

15 Neurochem 76, 1109-1120.

16 Smart, O.S., Neduvelil, J.G., Wang, X., Wallace, B.A., and Sansom, M.S. (1996). HOLE: a

17 program for the analysis of the pore dimensions of ion channel structural models. J Mol Graph

$18 \quad 14,354-360,376$.

19 Sola, M., Bavro, V.N., Timmins, J., Franz, T., Ricard-Blum, S., Schoehn, G., Ruigrok, R.W.,

20 Paarmann, I., Saiyed, T., O'Sullivan, G.A., et al. (2004). Structural basis of dynamic glycine

21 receptor clustering by gephyrin. The EMBO journal 23, 2510-2519. 
1 Sontheimer, H., Becker, C.M., Pritchett, D.B., Schofield, P.R., Grenningloh, G., Kettenmann, H.,

2 Betz, H., and Seeburg, P.H. (1989). Functional chloride channels by mammalian cell expression

3 of rat glycine receptor subunit. Neuron 2, 1491-1497.

4 Specht, C.G., Izeddin, I., Rodriguez, P.C., El Beheiry, M., Rostaing, P., Darzacq, X., Dahan, M.,

5 and Triller, A. (2013). Quantitative nanoscopy of inhibitory synapses: counting gephyrin

6 molecules and receptor binding sites. Neuron 79, 308-321.

7 Takahashi, T., Momiyama, A., Hirai, K., Hishinuma, F., and Akagi, H. (1992). Functional

8 correlation of fetal and adult forms of glycine receptors with developmental changes in

9 inhibitory synaptic receptor channels. Neuron 9, 1155-1161.

10 Usukura, E., Narita, A., Yagi, A., Ito, S., and Usukura, J. (2016). An Unroofing Method to

11 Observe the Cytoskeleton Directly at Molecular Resolution Using Atomic Force Microscopy. Sci

$12 \operatorname{Rep} 6,27472$.

13 Walker, P.A., Leong, L.E., Ng, P.W., Tan, S.H., Waller, S., Murphy, D., and Porter, A.G.

14 (1994). Efficient and rapid affinity purification of proteins using recombinant fusion proteases.

15 Biotechnology (N Y) 12, 601-605.

16 Wang, W., Touhara, K.K., Weir, K., Bean, B.P., and MacKinnon, R. (2016). Cooperative

17 regulation by $\mathrm{G}$ proteins and $\mathrm{Na}(+)$ of neuronal GIRK2 $\mathrm{K}(+)$ channels. Elife 5 .

18 Wang, W., Whorton, M.R., and MacKinnon, R. (2014). Quantitative analysis of mammalian

19 GIRK2 channel regulation by G proteins, the signaling lipid PIP2 and $\mathrm{Na}+$ in a reconstituted

$20 \quad$ system. eLife 3, e03671.

21 Wassle, H., Heinze, L., Ivanova, E., Majumdar, S., Weiss, J., Harvey, R.J., and Haverkamp, S.

22 (2009). Glycinergic transmission in the Mammalian retina. Frontiers in molecular neuroscience $232,6$. 
1 Weltzien, F., Puller, C., O'Sullivan, G.A., Paarmann, I., and Betz, H. (2012). Distribution of the

2 glycine receptor beta-subunit in the mouse CNS as revealed by a novel monoclonal antibody.

3 The Journal of comparative neurology 520, 3962-3981.

4 Werman, R., Davidoff, R.A., and Aprison, M.H. (1967). Inhibition of motoneurones by

5 iontophoresis of glycine. Nature 214, 681-683.

6 Williams, C.J., Headd, J.J., Moriarty, N.W., Prisant, M.G., Videau, L.L., Deis, L.N., Verma, V.,

7 Keedy, D.A., Hintze, B.J., Chen, V.B., et al. (2018). MolProbity: More and better reference data

8 for improved all-atom structure validation. Protein Sci 27, 293-315.

9 Yang, Z., Cromer, B.A., Harvey, R.J., Parker, M.W., and Lynch, J.W. (2007). A proposed

10 structural basis for picrotoxinin and picrotin binding in the glycine receptor pore. J Neurochem

$11 \quad 103,580-589$.

12 Yang, Z., Taran, E., Webb, T.I., and Lynch, J.W. (2012). Stoichiometry and subunit arrangement

13 of alpha1beta glycine receptors as determined by atomic force microscopy. Biochemistry 51,

$14 \quad 5229-5231$.

15 Yu, J., Zhu, H., Lape, R., Greiner, T., Du, J., Lu, W., Sivilotti, L., and Gouaux, E. (2021).

16

17

18

19

20

21

22

23
Mechanism of gating and partial agonist action in the glycine receptor. Cell.

Yu, T.W., Chahrour, M.H., Coulter, M.E., Jiralerspong, S., Okamura-Ikeda, K., Ataman, B.,

Schmitz-Abe, K., Harmin, D.A., Adli, M., Malik, A.N., et al. (2013). Using whole-exome sequencing to identify inherited causes of autism. Neuron 77, 259-273.

Zacchi, P., Antonelli, R., and Cherubini, E. (2014). Gephyrin phosphorylation in the functional organization and plasticity of GABAergic synapses. Frontiers in cellular neuroscience $8,103$.

Zacharias, D.A., Violin, J.D., Newton, A.C., and Tsien, R.Y. (2002). Partitioning of lipidmodified monomeric GFPs into membrane microdomains of live cells. Science 296, 913-916. 
1 Zeng, M., Chen, X., Guan, D., Xu, J., Wu, H., Tong, P., and Zhang, M. (2018). Reconstituted

2 Postsynaptic Density as a Molecular Platform for Understanding Synapse Formation and

3 Plasticity. Cell 174, 1172-1187 e1116.

4 Zhang, K. (2016). Gctf: Real-time CTF determination and correction. J Struct Biol 193, 1-12.

5 Zheng, S.Q., Palovcak, E., Armache, J.P., Verba, K.A., Cheng, Y.F., and Agard, D.A. (2017).

6 MotionCor2: anisotropic correction of beam-induced motion for improved cryo-electron

7 microscopy. Nature Methods 14, 331-332.

8 Zhu, S., Noviello, C.M., Teng, J., Walsh, R.M., Jr., Kim, J.J., and Hibbs, R.E. (2018). Structure

9 of a human synaptic GABAA receptor. Nature 559, 67-72. 
$5 \quad{ }^{1}$ Department of Biophysics, University of Texas Southwestern Medical Center, Dallas, TX

675390, USA

$7 \quad$ *Correspondence: Weiwei.Wang@UTSouthwestern.edu 
A

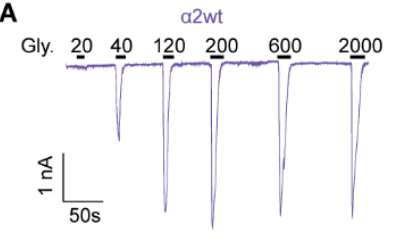

B

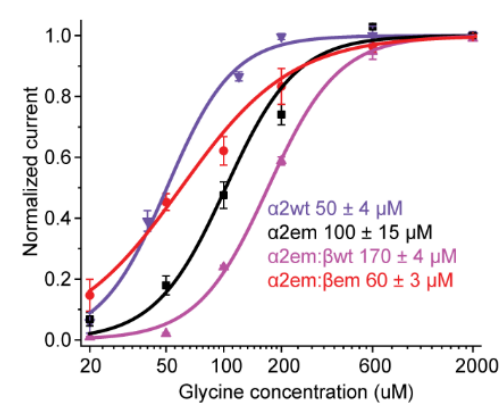

a2em

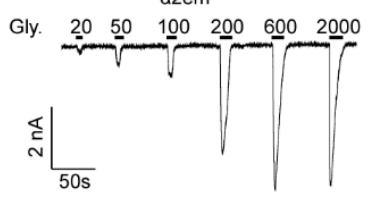

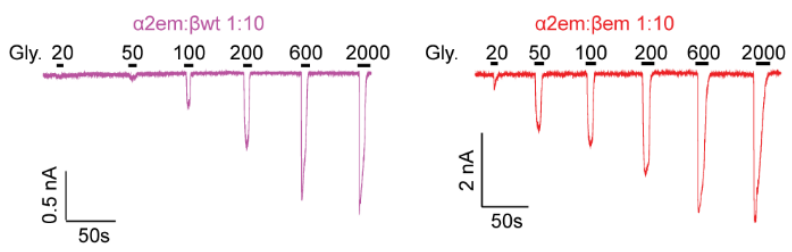

C

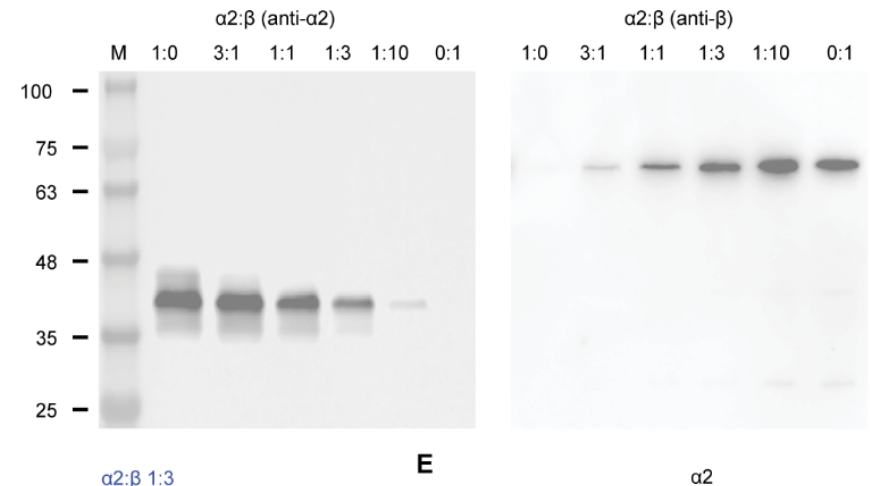

D
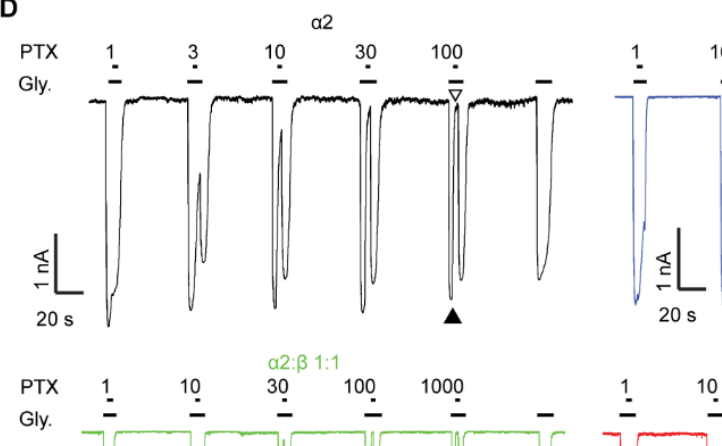

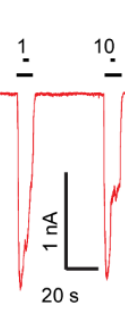

$\mathbf{F}$

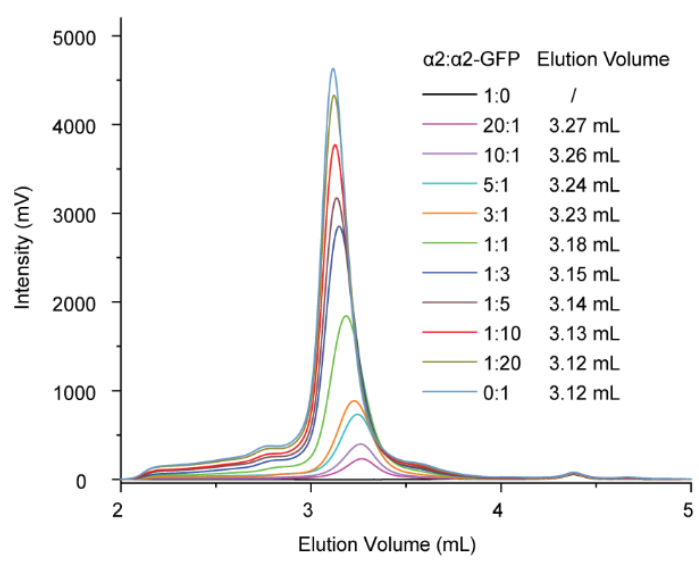

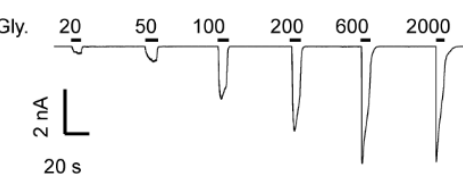

$20 \mathrm{~s}$

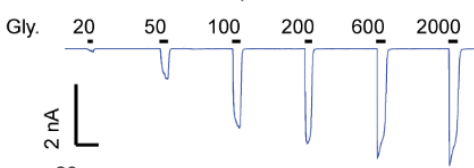

$20 \mathrm{~s}$

$\alpha 2: \beta 1: 10$
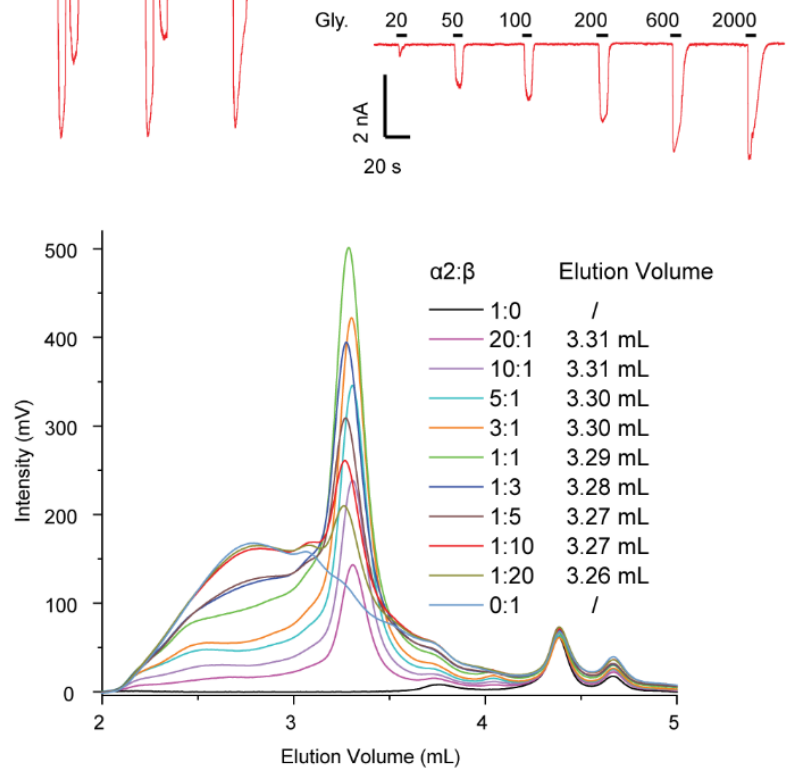

1

\section{GlyRs constructs, related to Figure 1.}


1 (A) Representative voltage-clamp recordings of glycine dose response in HEK293 cells transfected with $\alpha 2 \mathrm{wt}$ (magenta), $\alpha 2 \mathrm{em}$ (black), $\alpha 2 \mathrm{em}: \beta \mathrm{wt} 1: 10$ (pink) and $\alpha 2 \mathrm{em}: \beta \mathrm{em} 1: 10$ (red) DNAs.

4 (B) Dose response of glycine from (A). Data points with S.E.M. ( $\mathrm{n}=3-6$ cells) were plotted with $5 \quad$ Hill fits and colored the same as in (A). EC50 values are listed.

6 (C) Western blot of $\alpha 2$ and $\beta$ subunits at different DNA ratios. M: molecular weight marker,

7 molecular weights are in $\mathrm{kDa}$. See methods for details.

8 (D) PTX inhibition in the presence of $100 \mu \mathrm{M}$ glycine. $\alpha 2: \beta$ expression ratios of $1: 0,1: 1,1: 3$ and 9 1:10 were used. The PTX and glycine concentrations are in $\mu \mathrm{M}$.

10 (E) Glycine activation at $\alpha 2: \beta$ ratios of 1:0, 1:3 and 1:10. The glycine concentrations are in $\mu \mathrm{M}$.

11 (F) FSEC of $\alpha 2$ and $\alpha 2 \beta$ GlyRs at different $\alpha 2: \beta$ virus ratios. Left panel, FSEC of $\alpha 2$ GlyR

12 expressed at listed $\alpha 2: \alpha 2-T$ si-GFP virus ratios. Right panel, FSEC of $\alpha 2 \beta$ GlyR expressed at

13 different $\alpha 2: \beta$ virus ratios. Elution volumes are listed beside each virus ratio. Molecular weight

14 of $\alpha 2, \alpha 2-$ Tsi-GFP and $\beta$ are $45 \mathrm{KDa}, 87 \mathrm{KDa}$ and $77 \mathrm{KDa}$, respectively. 

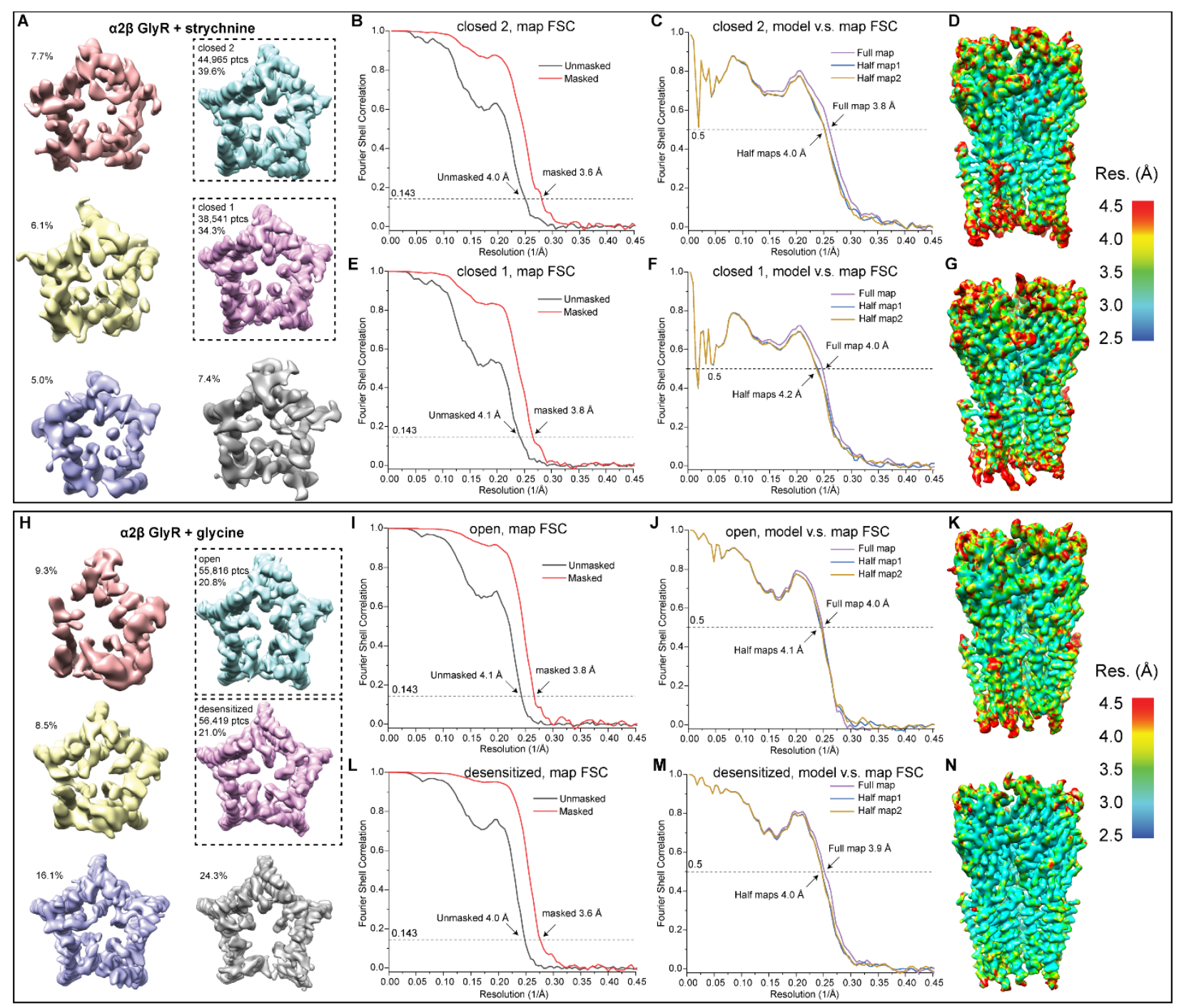

Figure S2. Classes of $\alpha 2 \beta$ GlyR density maps, related to Figure 3.

3 (A-G) $\alpha 2 \beta$ GlyR closed states. (A) 6 classes resulting from 3D classification after signalsubtraction view along the ion conduction pore from the intracellular side. The percentages of particles in each class are indicated. The two good classes were subsequently refined. (B) The gold standard Fourier Shell Correlation (FSC) curves from the final 3D refinement of closed state 2, with (red) and without (black) mask. (C) FSC between the atomic model and half map 1 (blue), half map 2 (orange) and the full map (purple) of closed state 2. (D) Density map of closed state 2 colored according to its local resolution estimated using RESMAP. (E) The gold standard 
$1 \quad$ FSC from the final map of closed state 1. (F) FSC between the atomic model and maps of closed

2 state 2. Color codes are the same as in (B) and (C). (G) Density map of closed state 1 colored

3 according to its local resolution estimated using RESMAP.

$4 \quad(\mathrm{H}-\mathrm{N}) \alpha 2 \beta$ GlyR open state and desensitized state. (H) 6 classes resulting from 3D classification.

5 The two good classes were subsequently refined. (I-J) FSC curves of open states. (K) Density

6 map of open state colored according to its local resolution (L-M) FSC curves of desensitized

7 states. (N) Density map of open state colored according to its local resolution. 
A

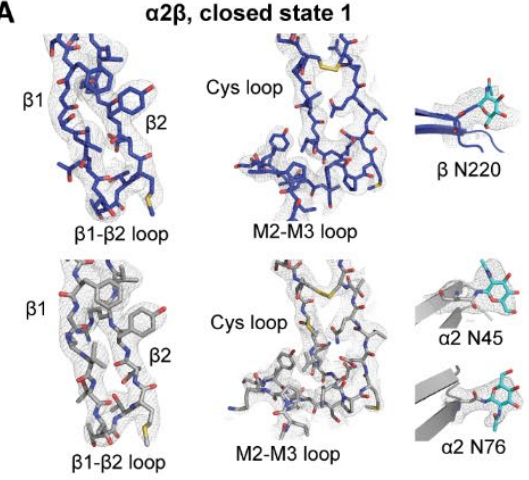

C

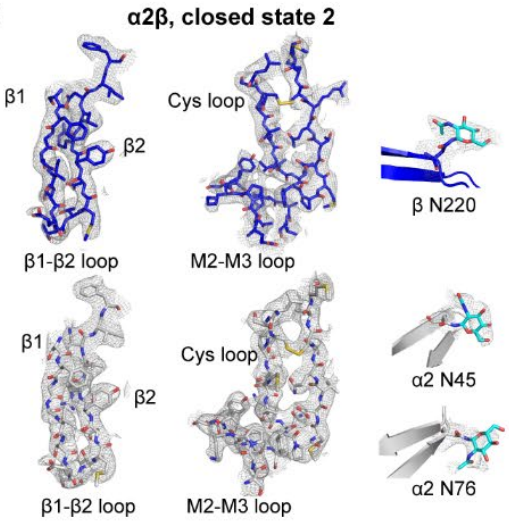

$\mathbf{E}$

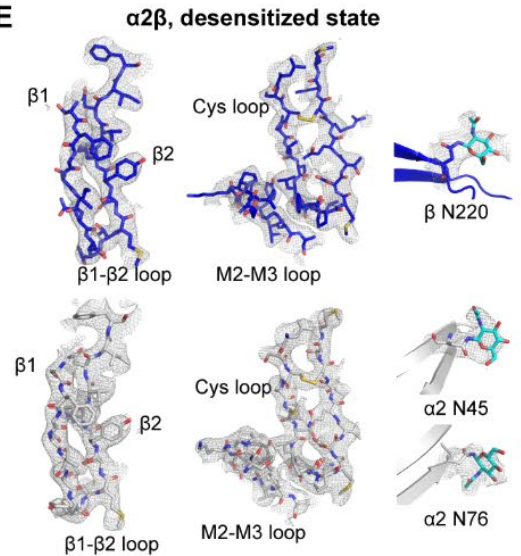

G

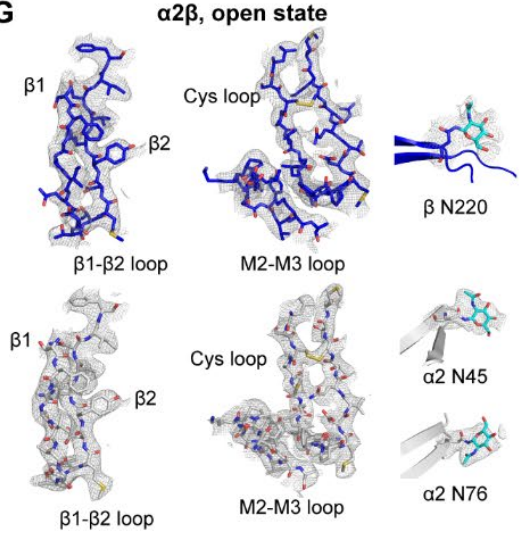

B

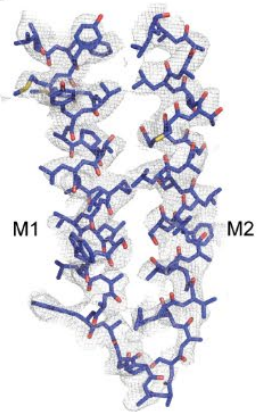

D

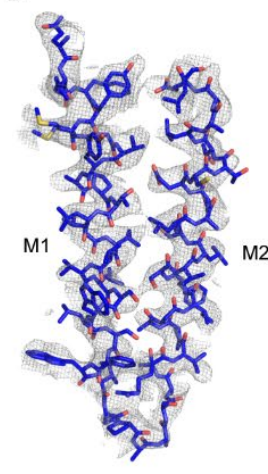

$\mathbf{F}$

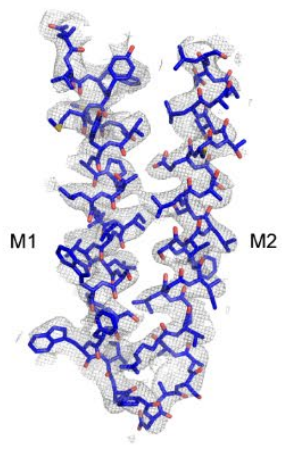

H

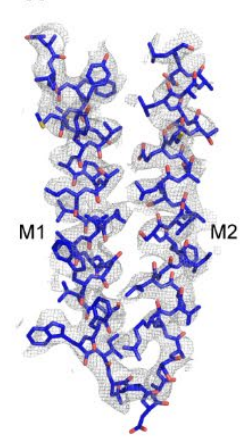

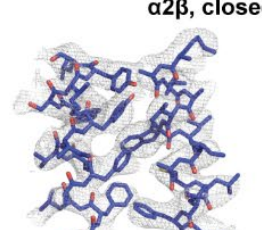
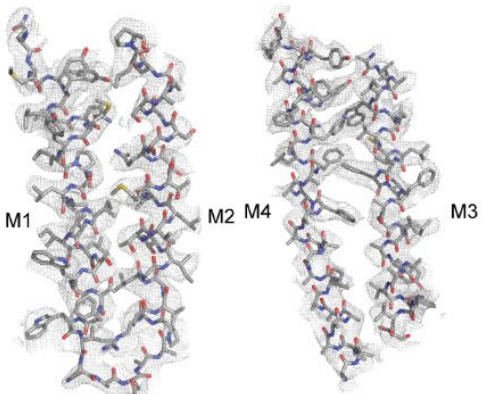

$\alpha 2 \beta$, closed state 2

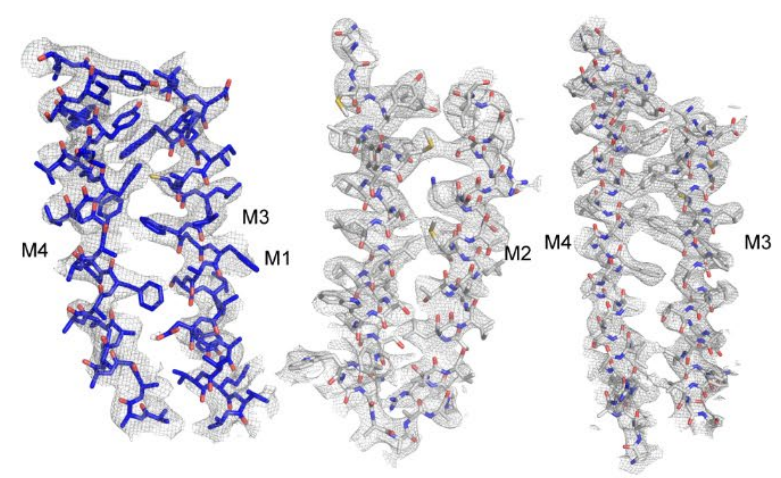

$\alpha 2 \beta$, desensitized state

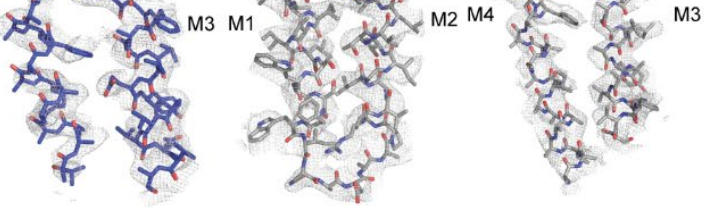

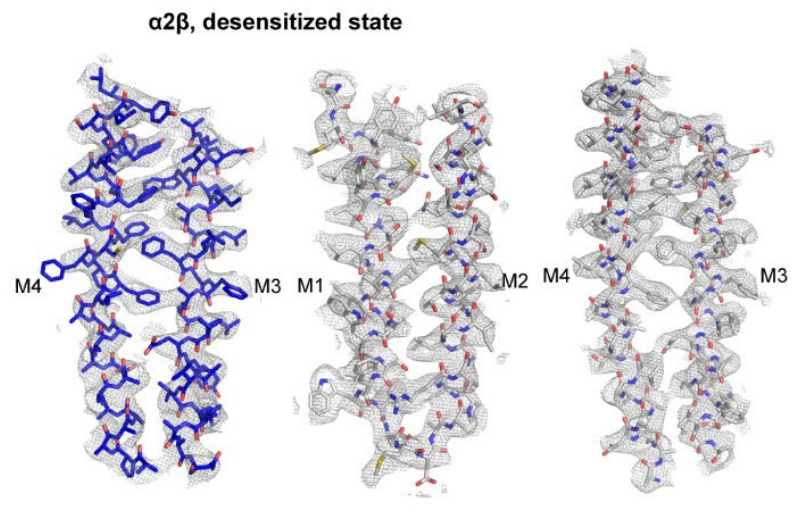

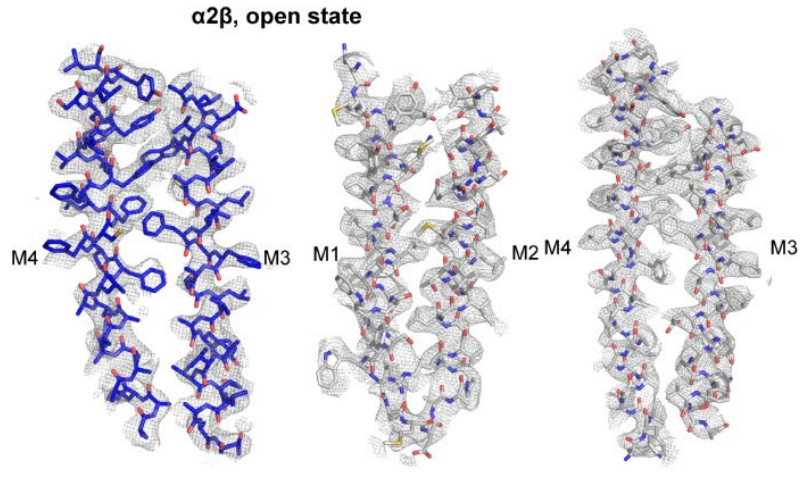

Figure S3. Typical density maps, related to Figure 3 and Figure 4. 
1 (A-B) Densities maps of representative regions of $\alpha 2 \beta$ GlyR closed state 1. (A) Density maps of

$2 \quad \beta$-sheet, Cys-loop and M2-M3 loop in $\beta$ subunit ECD; N-glycosylation on the $\beta$ subunit ECD; $\beta$ -

3 sheet, Cys-loop and M2-M3 loop in $\alpha 2$ subunit ECD; Two N-glycosylation sites on $\alpha 2$ subunit

4 ECD. (B) M1-M4 Helices of $\beta$ subunit and $\alpha 2$ subunit TMD.

5 (C-D) Densities maps of representative regions of $\alpha 2 \beta$ GlyR closed state 2 . Regions are the same

6 as (A-B).

7 (E-F) Densities maps of representative regions of $\alpha 2 \beta$ GlyR desensitized state. Regions are the

8 same as (A-B).

9 (G-H) Densities maps of representative regions of $\alpha 2 \beta$ GlyR open state. Regions are the same as $10 \quad(\mathrm{~A}-\mathrm{B})$. 


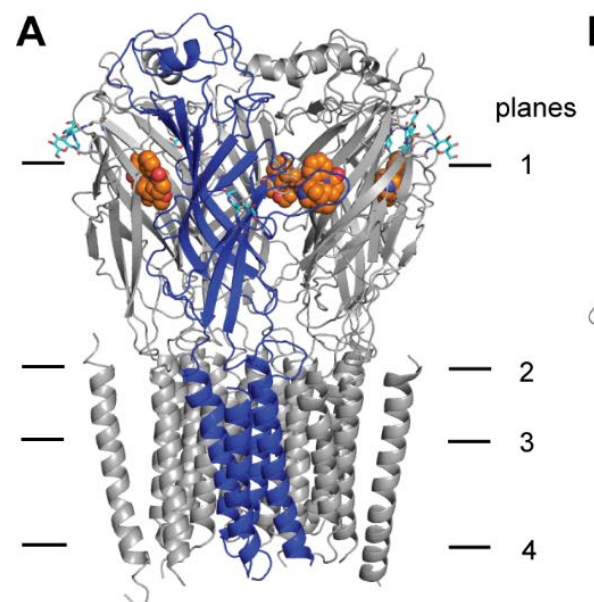

B lanes

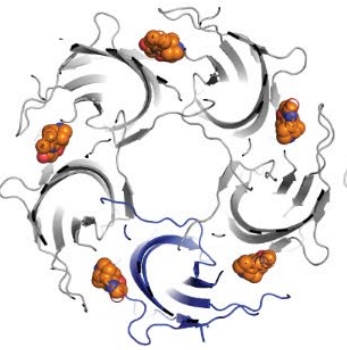

strychnine, closed $1 / 2$

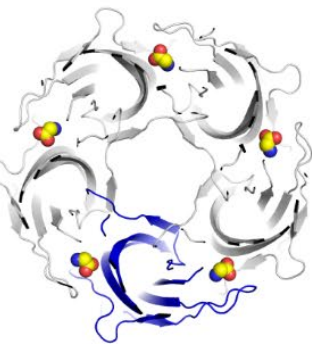

glycine, desensitized

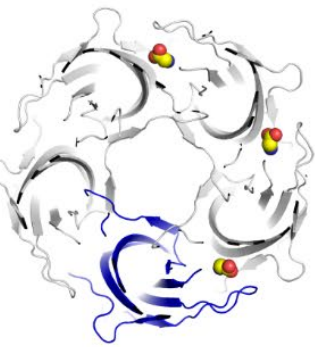

glycine, open
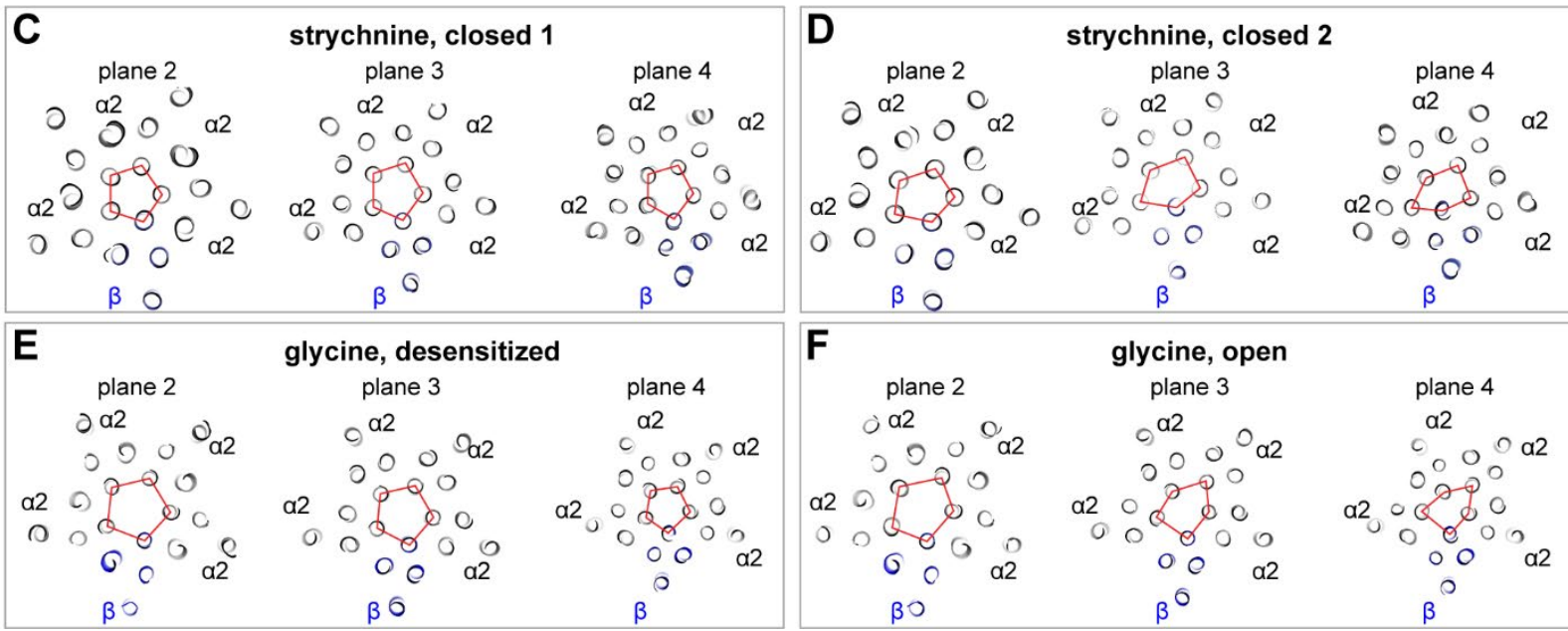

\section{glycine, open}

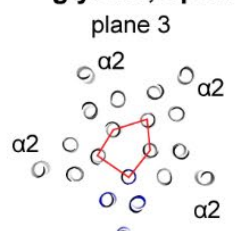
$\beta^{6}$

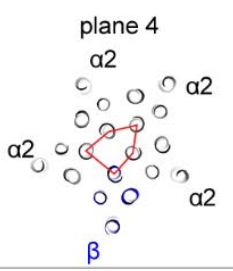

Figure S4. Symmetry of the $\alpha 2 \beta$ GlyR, related to Figure 3.

(A) $\alpha 2 \beta$ GlyR closed state 2 viewed parallel to the membrane with four planes indicated.

(B) Top views from the extracellular side clipped at plane 1 of closed, desensitized, and open states.

(C) Top view of planes 2-4 for closed state 1.

(D) Top view of planes 2-4 for closed state 2 .

(E) Top view of planes 2-4 for desensitized state.

(F) Top view of planes 2-4 for open state. $\alpha 2$ subunit is colored in gray, $\beta$ in blue, strychnine in

11 pentagons in panels $(\mathrm{C}-\mathrm{F})$ indicate the $\mathrm{M} 2$ helices positions. 

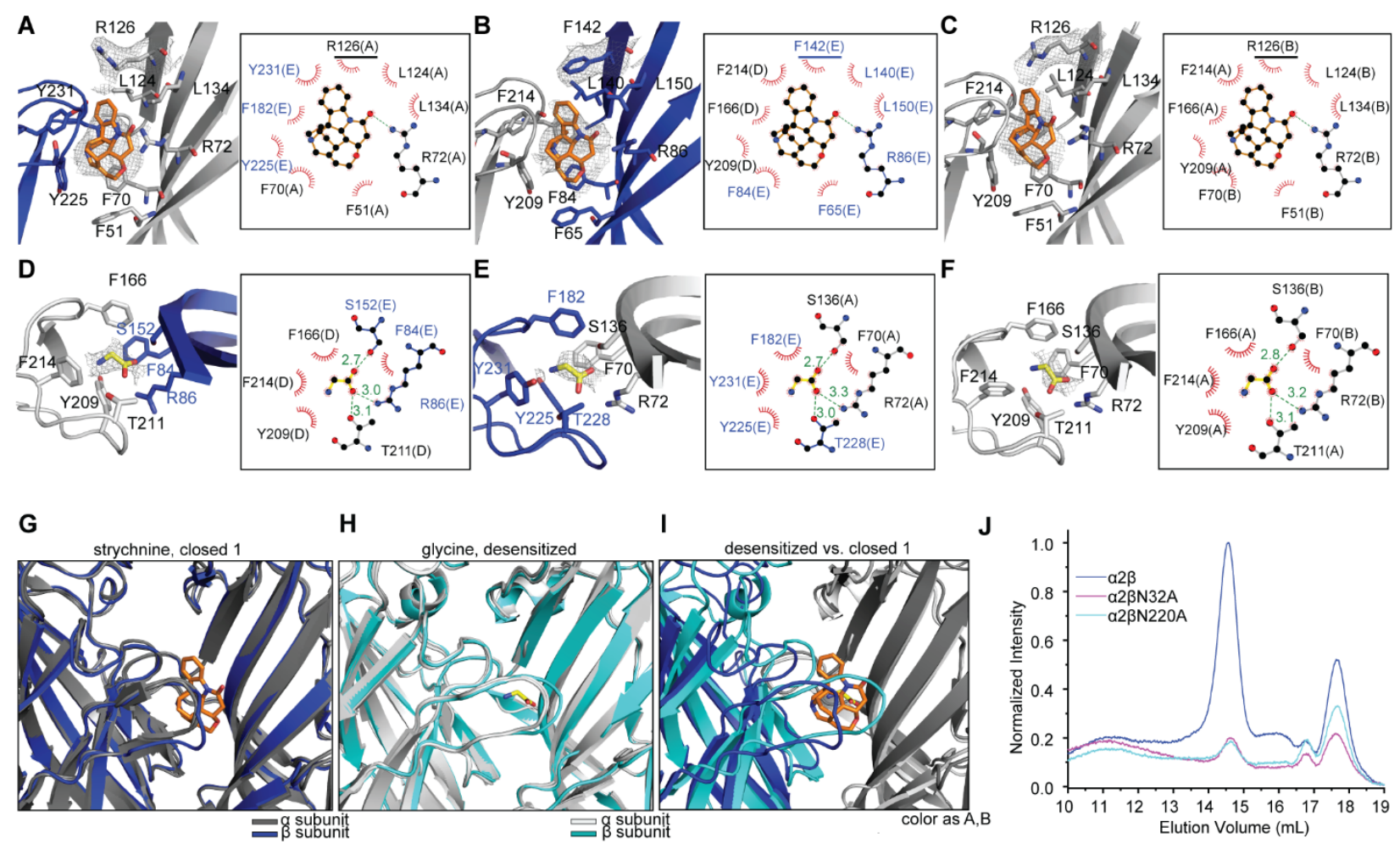

Figure S5. Strychnine and glycine binding sites, related to Figure 3 and Figure 4.
(A-C) Strychnine binding sites between (A) $\beta / \alpha 2$, (B) $\alpha 2 / \beta$ and (C) $\alpha 2 / \alpha 2$ interfaces from closed

state 2. Densities of $\alpha 2$ R126, $\beta$ F142 and Strychnine are contoured at $3 \sigma$. Strychnine (orange)

and the residues interacting with strychnine ( $\alpha 2$ : grey, $\beta$ : blue) are shown as sticks. LigPlot

schematic of strychnine bind sites between (A) $\beta / \alpha 2$, (B) $\alpha 2 / \beta$ and (C) $\alpha 2 / \alpha 2$ interfaces, showing

electrostatic (dashes) and hydrophobic (eyelashes) interactions. Non-conservative residues $\alpha 2$

R126 and $\beta$ F142 are underlined.

(D-F) Glycine binding sites between (D) $\alpha 2 / \beta$, (E) $\beta / \alpha 2$ and (F) $\alpha 2 / \alpha 2$ interfaces from

desensitized state. Densities of glycine are contoured at $4 \sigma$. Glycine (yellow) and the residues

interacting with glycine ( $\alpha 2$ : grey, $\beta$ : blue) are shown as sticks. LigPlot schematic of glycine bind sites between (D) $\alpha 2 / \beta$, (E) $\beta / \alpha 2$ and (F) $\alpha 2 / \alpha 2$ interfaces, showing electrostatic (dashes) and hydrophobic (eyelashes) interactions. 
1 (G) Strychnine binding sites between $\beta / \alpha 2, \alpha 2 / \beta$ and $\alpha 2 / \alpha 2$ interfaces from closed state 1 .

2 Strychnine (carbon as orange, nitrogen as blue and oxygen as red) are shown as sticks, $\alpha 2$ and $\beta$

3 ( $\alpha 2$ : grey, $\beta$ : blue) are shown as cartoon.

4 (H) Glycine binding sites between $\beta / \alpha 2, \alpha 2 / \beta$ and $\alpha 2 / \alpha 2$ interfaces from desensitized state.

5 Glycine (carbon as yellow, nitrogen as blue and oxygen as red) are shown as sticks, $\alpha 2$ and $\beta$

$6 \quad(\alpha 2$ : grey, $\beta$ : cyan) are shown as cartoon.

7 (I) Overlap of glycine/strychnine binding site between $\beta / \alpha 2$ interface from desensitized/closed 1

8 state. The colors are same as $(\mathrm{G})$ and $(\mathrm{H})$.

9 (J) FSEC of $\alpha 2 \beta$ GlyR with glycosylation site mutation. Wild type $\alpha 2 \beta$ are colored as blue, $\alpha 2 \beta$

10 with $\beta$ :N32A mutation are colored as pink, $\alpha 2 \beta$ with $\beta$ :N220A mutation are colored as cyan. 
bioRxiv preprint doi: https://doi.org/10.1101/2021.05.17.444520; this version posted May 18, 2021. The copyright holder for this preprint (which was not certified by peer review) is the author/funder. All rights reserved. No reuse allowed without permission.
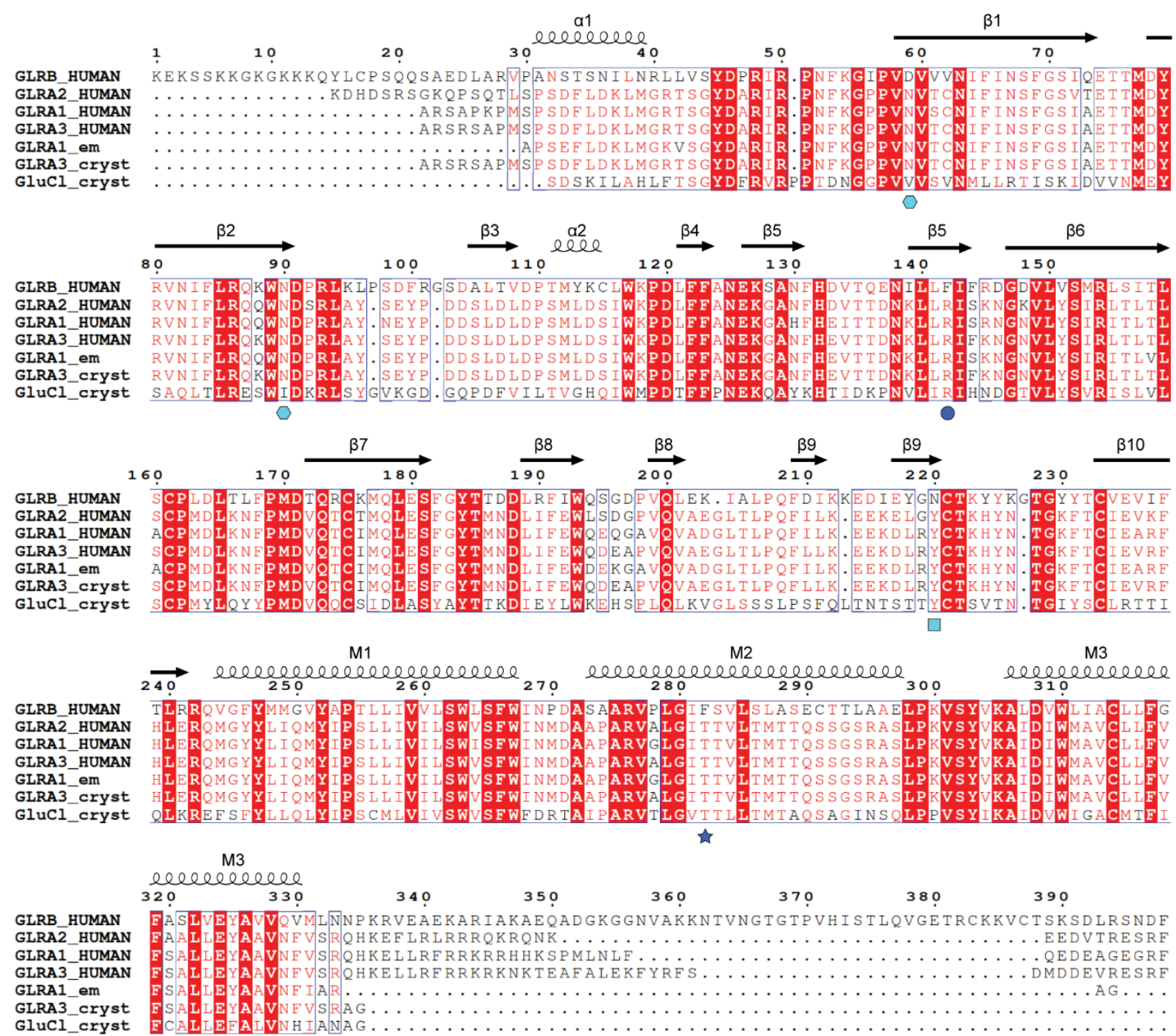

GluCl_cryst

GLRB_HUMAN
GLRA2_HUMAN
GLRA1_HUMAN
GLRA3_HUMAN
GLRA1_em
GLRA3_cryst
GluC1_cryst

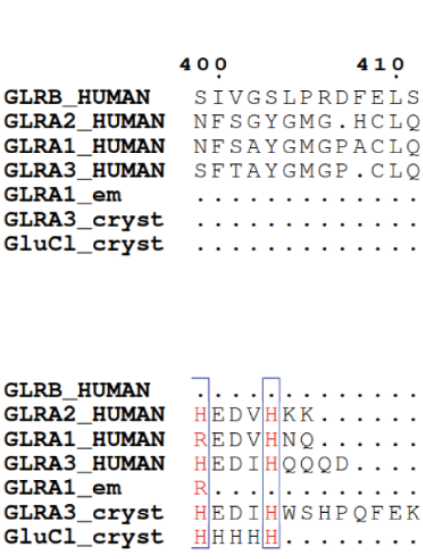

2 Figure S6. Sequence alignment of GlyR subunits, related to Figure 4. 
1 Signal peptides have been removed before sequence alignment. Residue conservation is

2 indicated by blue rectangle and red highlights. Secondary structure elements are indicated by

3 helices for $\alpha$-helices and arrows for $\beta$-strands above the alignment. $N$-linked glycosylation site of

4 GlyR $\alpha 2$ and $\beta$ are indicated by cyan hexagon and cyan rectangle, respectively. Non-conservative

5 residue between GlyR $\alpha 2$ and $\beta$ involved in strychnine binding is indicated by blue circle. The

6 Phe282 in M2 helix of GlyR $\beta$ is indicated by blue star. The alignment was generated by Clustal

7 W. Protein sequences alignment: human GLRB (Uniprot P48167), human GLRA2 (Uniprot

8 P23416), human GLRA1 (Uniprot P23415), human GLRA3 (Uniprot O75311), GLRA1em(PDB

9 accession number 3JAE), GLRA3cryst (PDB accession number 5CFB) and GluClcryst (PDB

10 accession number 4TNV). 


\section{Table S1.}

Cryo-EM data collection, refinement and validation statistics

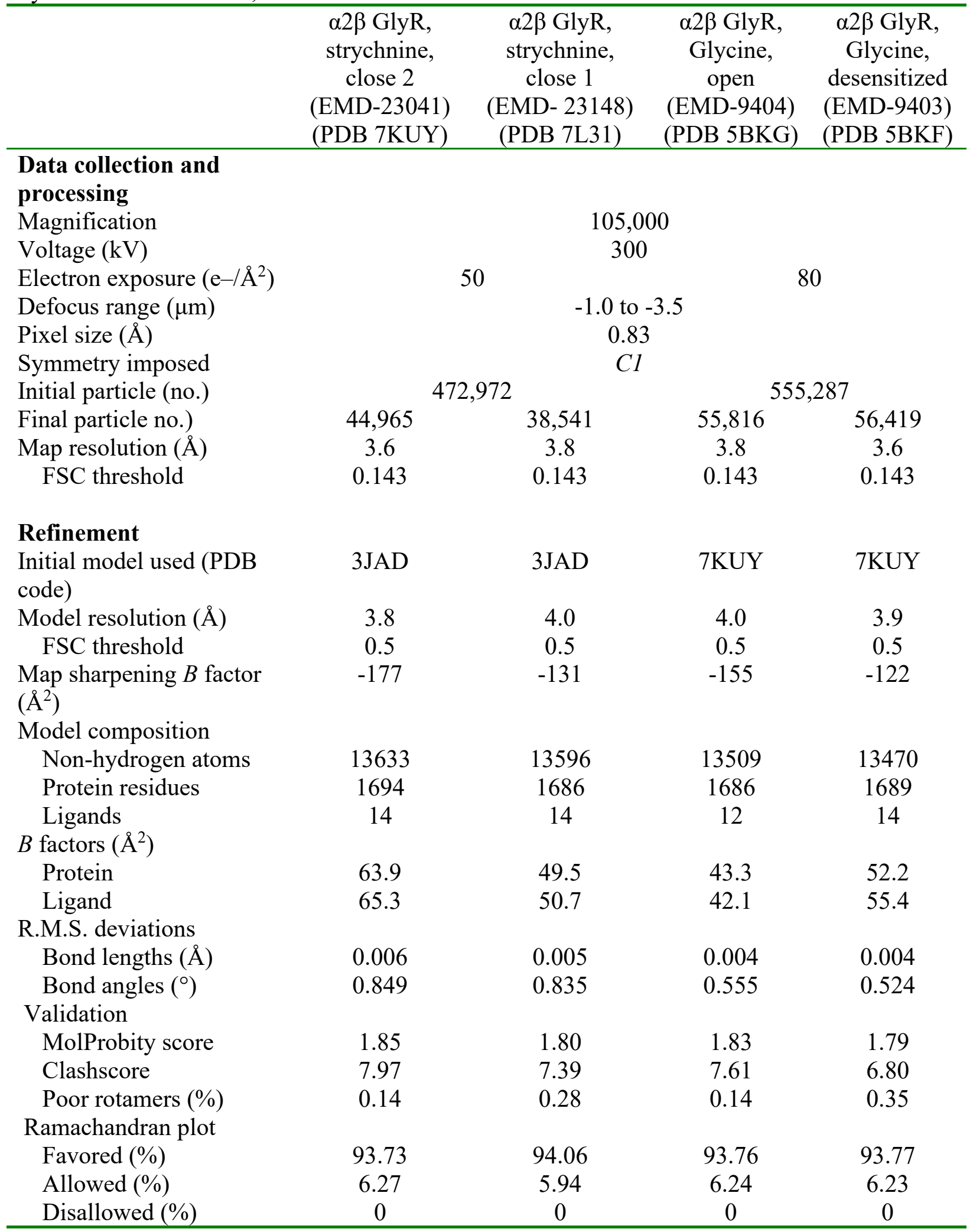


bioRxiv preprint doi: https://doi.org/10.1101/2021.05.17.444520; this version posted May 18, 2021. The copyright holder for this preprint (which was not certified by peer review) is the author/funder. All rights reserved. No reuse allowed without permission. 